\title{
Compactness Properties for Modulation Spaces
}

\author{
Christine Pfeuffer ${ }^{1}$. Joachim Toft ${ }^{2}$
}

Received: 3 October 2018 / Accepted: 12 February 2019 / Published online: 9 March 2019

(c) The Author(s) 2019

\section{Abstract}

We prove that if $\omega_{1}$ and $\omega_{2}$ are moderate weights and $\mathscr{B}$ is a suitable (quasi-)Banach function space, then a necessary and sufficient condition for the embedding $i$ : $M\left(\omega_{1}, \mathscr{B}\right) \rightarrow M\left(\omega_{2}, \mathscr{B}\right)$ between two modulation spaces to be compact is that the quotient $\omega_{2} / \omega_{1}$ vanishes at infinity. Moreover we show, that the boundedness of $\omega_{2} / \omega_{1}$ is a necessary and sufficient condition for the previous embedding to be continuous.

Keywords Gelfand-Shilov spaces · Distributions · Bargmann transform . Quasi-Banach spaces

Mathematics Subject Classification 42B35 $\cdot 46 \mathrm{~B} 50 \cdot 54 \mathrm{D} 30 \cdot 46 \mathrm{Fxx}$

\section{Introduction}

A fundamental question in analysis, science and engineering concerns compactness. In the paper we investigate continuity and compactness properties for a broad class of modulation spaces, a family of functions and distribution spaces on the configuration space $\mathbf{R}^{d}$, introduced by Feichtinger [12]. The basic theory of these spaces was thereafter established by Feichtinger and Gröchenig (see [15,16,25] and the references therein).

A modulation space is defined by imposing a certain norm or quasi-norm estimate on the short-time Fourier transform of the involved functions and distributions. (See [28] or Sect. 2 for definitions and notations.) Roughly speaking, this means that each

Communicated by Palle Jorgensen.

$凶$ Joachim Toft

joachim.toft@lnu.se

Christine Pfeuffer

christine.pfeuffer@mathematik.uni-regensburg.de

1 Department of Mathematics, University of Regensburg, Regensburg, Germany

2 Department of Mathematics, Linnæus University, Växjö, Sweden

Dirkhäuser 
modulation space norm measures in a certain way the phase or time-frequency content. That is, it admits to measure the configuration energy and momentum energy simultaneously, in a certain way.

By the design of these spaces it turns out that they are useful in several fields in analysis, physics and engineering (see $[6,8,31,34]$ and the references therein).

Compactness investigations of embeddings between modulation spaces go in some sense back to [32], where M. Shubin proved that if $t>0$, then the embedding $i$ : $Q_{s} \rightarrow Q_{s-t}$ is compact. In the community, the previous compactness property was not obvious because similar facts do not hold for Sobolev spaces of Hilbert type. That is, for $t>0$ it is well-known that the embedding $i: H_{s}^{2} \rightarrow H_{s-t}^{2}$ is continuous but not compact. Since

$$
Q_{s}=M_{(\omega)}^{2,2}, \quad \omega(X)=(1+|x|+|\xi|)^{S}
$$

and

$$
H_{s}^{2}=M_{(\omega)}^{2,2}, \quad \omega(X)=(1+|\xi|)^{s}, \quad X=(x, \xi)
$$

the previous compact embedding properties can also be written by means of modulation spaces. A more general situation was considered by M. Dörfler, H. Feichtinger and K. Gröchenig who considered the inclusion map

$$
i: M_{\left(\omega_{1}\right)}^{p, q}\left(\mathbf{R}^{d}\right) \rightarrow M_{\left(\omega_{2}\right)}^{p, q}\left(\mathbf{R}^{d}\right)
$$

in [9]. It is well-known that this map is well-defined and continuous when $\omega_{2} \lesssim \omega_{1}$, see e.g. [25]. In [9, Theorem 5] it was proved that if $p, q \in[1, \infty)$, and $\omega_{1}$ and $\omega_{2}$ are certain moderate weights of polynomial type, then (1.3) is compact if and only if $\omega_{2} / \omega_{1}$ tends to zero at infinity. By choosing $\omega_{j}$ in similar ways as in (1.1), the latter compactness result confirms the compactness of the embedding $i: Q_{s} \rightarrow Q_{s-t}$ above by Shubin, as well as it confirms the lack of compactness of the embedding $i: H_{s}^{2} \rightarrow H_{s-t}^{2}$ for Sobolev spaces.

In [3], the compact embedding property [9, Theorem 5] was extended in such a way that all moderate weights $\omega_{j}$ of polynomial type are included. That is, there are no other restrictions on $\omega_{j}$ than there should exist constants $N_{j}>0$ such that

$$
\omega_{j}(X+Y) \lesssim \omega_{j}(X)(1+|Y|)^{N_{j}}, \quad X, Y \in \mathbf{R}^{2 d}, \quad j=1,2
$$

Moreover, in [3], the Lebesgue exponents $p$ and $q$ in the modulation spaces are allowed to attain $\infty$.

In our main results, Theorems 3.7 and 3.9 in Sect. 3, these continuity and compactness results are extended to involve modulation spaces $M(\omega, \mathscr{B})$, which are more general in different ways. Firstly, there are no boundedness estimates of polynomial type for the involved weight $\omega$. In most of our considerations, we require that the weights are moderate, which implies that the condition (1.4) is relaxed into

$$
\omega_{j}(X+Y) \lesssim \omega_{j}(X) e^{r_{j}|Y|}, \quad j=1,2
$$


for some constants $r_{1}, r_{2}>0$.

Secondly, $\mathscr{B}$ can be any general translation invariant Banach function space without the restriction that $M(\omega, \mathscr{B})$ should be of the form $M_{(\omega)}^{p, q}$. We may also have $M(\omega, \mathscr{B})=M_{(\omega)}^{p, q}$, but in contrast to [3,9], we here allow $p$ and $q$ to be smaller than 1 . Here we notice that if $p<1$ or $q<1$, then $M_{(\omega)}^{p, q}$ fails to be a Banach space because of absence of convex topological structures.

Thirdly, we show that (1.3) is compact when $\omega_{2} / \omega_{1}$ tends to zero at infinity, when the assumptions on $\omega_{1}$ and $\omega_{2}$ are relaxed into a suitable local moderate condition (cf. Theorem 3.9(1) in Sect. 3). We refer to [40] and to some extent to [42] for a detailed study of modulation spaces with such relaxed conditions on the involved weight functions.

In Sect. 4 we show how Theorem 3.9 has been applied in [1] to deduce index results and lifting properties for certain pseudo-differential operators. Especially it is here indicated in which way our results on compactness are used to show that the operator $\mathrm{Op}_{A}\left(\omega_{0}\right)$ is continuous and bijective from $M_{(\omega)}^{p, q}$ to $M_{\left(\omega / \omega_{0}\right)}^{p, q}$ when

$$
\omega_{0}(x, \xi) \equiv p_{1}(x)^{r}+p_{2}(\xi)^{\rho}
$$

$r, \rho>0$, and $p_{1}$ and $p_{2}$ are positive polynomials on $\mathbf{R}^{d}$.

In Sect. 4 we also give some links on possible extensions and generalizations.

Finally we remark that for moderate weights, the continuity and compactness properties for (1.3) can also be obtained by Gabor analysis, which transfers (1.3) into

$$
i: \ell_{\left(\omega_{1}\right)}^{p, q} \rightarrow \ell_{\left(\omega_{2}\right)}^{p, q}
$$

Since it is clear that the latter inclusion map is compact, if and only if $\omega_{2} / \omega_{1}$ tends to 0 at infinity, it follows that the compactness results in $[3,32]$ as well as some of the results in Sect. 3 can be deduced in such ways. We emphasise however that such technique can not be used in those situations in Sect. 3 when modulation spaces are of the form $M(\omega, \mathscr{B})$, where either $\mathscr{B}$ is a general BF-space, or $\omega$ fails to be moderate, since it seems that Gabor analysis is not applicable on such modulation spaces.

Parts of the proof of these continuity and compactness results are based on some properties for modulation spaces which might be of independent interests. We prove that if $\mathscr{B}$ is a Banach Function space, then $M(\omega, \mathscr{B})$ is a Banach space, which is continuously embedded in a weighted modulation space of the form $M_{(1 / v)}^{\infty}\left(\mathbf{R}^{d}\right)$ for certain weights $v$. (See Proposition 3.2.) We also need some properties for the Bargmann transform when acting on modulation spaces, essentially deduced in $[40,42]$, which are presented in Sect. 2.5.

\section{Preliminaries}

In this section we discuss basic properties for modulation spaces and other related spaces. The proofs are in many cases omitted since they can be found in [10-12,1517,25,36-39]. 


\subsection{Weight Functions}

A weight or weight function $\omega$ on $\mathbf{R}^{d}$ is a positive function such that $\omega, 1 / \omega \in$ $L_{\text {loc }}^{\infty}\left(\mathbf{R}^{d}\right)$. Let $\omega$ and $v$ be weights on $\mathbf{R}^{d}$. Then $\omega$ is called $v$-moderate or moderate, if

$$
\omega\left(x_{1}+x_{2}\right) \lesssim \omega\left(x_{1}\right) v\left(x_{2}\right), \quad x_{1}, x_{2} \in \mathbf{R}^{d} .
$$

Here $f(\theta) \lesssim g(\theta)$ means that $f(\theta) \leq c g(\theta)$ for some constant $c>0$ which is independent of $\theta$ in the domain of $f$ and $g$. If $v$ can be chosen as a polynomial, then $\omega$ is called a weight of polynomial type.

The function $v$ is called submultiplicative, if it is even and (2.1) holds for $\omega=v$. We notice that (2.1) implies that if $v$ is submultiplicative on $\mathbf{R}^{d}$, then there is a constant $c>0$ such that $v(x) \geq c$ when $x \in \mathbf{R}^{d}$.

We let $\mathscr{P}_{E}\left(\mathbf{R}^{d}\right)$ be the set of all moderate weights on $\mathbf{R}^{d}$, and $\mathscr{P}\left(\mathbf{R}^{d}\right)$ be the subset of $\mathscr{P}_{E}\left(\mathbf{R}^{d}\right)$ which consists of all polynomially moderate functions on $\mathbf{R}^{d}$. For $s>0$, also let $\mathscr{P}_{E, s}\left(\mathbf{R}^{d}\right)\left(\mathscr{P}_{E, s}^{0}\left(\mathbf{R}^{d}\right)\right)$ be the set of all weights $\omega$ in $\mathbf{R}^{d}$ such that

$$
\omega\left(x_{1}+x_{2}\right) \lesssim \omega\left(x_{1}\right) e^{r\left|x_{2}\right|^{\frac{1}{s}}}, \quad x_{1}, x_{2} \in \mathbf{R}^{d}
$$

for some $r>0$ (for every $r>0$ ), and set $\mathscr{P}_{E}^{0}=\mathscr{P}_{E, 1}^{0}$. We have

$$
\mathscr{P} \subseteq \mathscr{P}_{E, s_{1}}^{0} \subseteq \mathscr{P}_{E, s_{1}} \subseteq \mathscr{P}_{E, s_{2}}^{0} \subseteq \mathscr{P}_{E} \quad \text { when } s_{2}<s_{1}
$$

and

$$
\mathscr{P}_{E, s}=\mathscr{P}_{E} \quad \text { when } s \leq 1 \text {, }
$$

where the last equality follows from the fact that if $\omega \in \mathscr{P}_{E}\left(\mathbf{R}^{d}\right)\left(\omega \in \mathscr{P}_{E}^{0}\left(\mathbf{R}^{d}\right)\right)$, then

$$
\omega(x+y) \lesssim \omega(x) e^{r|y|^{\frac{1}{s}}} \text { and } e^{-r|x|} \leq \omega(x) \lesssim e^{r|x|}, \quad x, y \in \mathbf{R}^{d}
$$

hold true for some $r>0$ (for every $r>0$ ) in view of [26] when $\mathrm{s} \leq 1$.

In some situations we shall consider a more general class of weights compared to $\mathscr{P}_{E}$ given in [40, Definition 1.1].

Definition 2.1 The set $\mathscr{P}_{Q}\left(\mathbf{R}^{d}\right)$ consists of all weights $\omega$ on $\mathbf{R}^{d}$ such that for some constants $R \geq 2$ and $c, r>0$ it holds

$$
e^{-r|x|^{2}} \lesssim \omega(x) \lesssim e^{r|x|^{2}}
$$

and

$$
\omega(x)^{2} \lesssim \omega(x+y) \omega(x-y) \lesssim \omega(x)^{2} \text { when } \quad R c \leq|x| \leq \frac{c}{|y|}
$$




\subsection{Gelfand-Shilov Spaces}

Let $0<h, s \in \mathbf{R}$. Then we denote the set of all functions $f \in C^{\infty}\left(\mathbf{R}^{d}\right)$ such that

$$
\|f\|_{\mathcal{S}_{s, h}} \equiv \sup \frac{\left|x^{\beta} \partial^{\alpha} f(x)\right|}{h^{|\alpha+\beta|}(\alpha ! \beta !)^{s}}<\infty
$$

by $\mathcal{S}_{s, h}\left(\mathbf{R}^{d}\right)$. Here the supremum is taken over all $\alpha, \beta \in \mathbf{N}^{d}$ and $x \in \mathbf{R}^{d}$.

It is clear that $\mathcal{S}_{s, h}$ is a Banach space which increases with $h$ and $s$, and that $\mathcal{S}_{s, h} \hookrightarrow \mathscr{S}$ holds. Here $A \hookrightarrow B$ means that $A \subseteq B$ with continuous embedding. If $s>\frac{1}{2}$, then

$$
\mathcal{S}_{s, h} \text { and } \bigcup_{h>0} S_{1 / 2, h}
$$

contains all finite linear combinations of the Hermite functions. By the density of such linear combinations in $\mathscr{S}$ and in $\mathcal{S}_{s, h}$, the dual $\mathcal{S}_{s, h}^{\prime}\left(\mathbf{R}^{d}\right)$ of $\mathcal{S}_{s, h}\left(\mathbf{R}^{d}\right)$ is a Banach space which contains $\mathscr{S}^{\prime}\left(\mathbf{R}^{d}\right)$, for such choices of $s$.

The inductive and projective limits respectively of $\mathcal{S}_{s, h}\left(\mathbf{R}^{d}\right)$ are called GelfandShilov spaces of Beurling respectively Roumieu type and are denoted by $\mathcal{S}_{S}\left(\mathbf{R}^{d}\right)$ and $\Sigma_{s}\left(\mathbf{R}^{d}\right)$. Hence

$$
\mathcal{S}_{S}\left(\mathbf{R}^{d}\right)=\bigcup_{h>0} \mathcal{S}_{s, h}\left(\mathbf{R}^{d}\right) \quad \text { and } \quad \Sigma_{s}\left(\mathbf{R}^{d}\right)=\bigcap_{h>0} \mathcal{S}_{s, h}\left(\mathbf{R}^{d}\right),
$$

where the topology for $\mathcal{S}_{S}\left(\mathbf{R}^{d}\right)$ is the strongest possible one such that the inclusion map from $\mathcal{S}_{s, h}\left(\mathbf{R}^{d}\right)$ to $\mathcal{S}_{S}\left(\mathbf{R}^{d}\right)$ is continuous, for every choice of $h>0$. Equipped with the seminorms $\|\cdot\|_{\mathcal{S}_{s, h}}, h>0$ the space $\Sigma_{s}\left(\mathbf{R}^{d}\right)$ is a Fréchet space. Additionally, $\Sigma_{s}\left(\mathbf{R}^{d}\right) \neq\{0\}$, if and only if $s>\frac{1}{2}$, and $\mathcal{S}_{S}\left(\mathbf{R}^{d}\right) \neq\{0\}$, if and only if $s \geq \frac{1}{2}$.

The Gelfand-Shilov distribution spaces $\mathcal{S}_{S}^{\prime}\left(\mathbf{R}^{d}\right)$ and $\Sigma_{S}^{\prime}\left(\mathbf{R}^{d}\right)$ are the projective and inductive limit respectively of $\mathcal{S}_{s, h}^{\prime}\left(\mathbf{R}^{d}\right)$. This implies that

$$
\mathcal{S}_{S}^{\prime}\left(\mathbf{R}^{d}\right)=\bigcap_{h>0} \mathcal{S}_{s, h}^{\prime}\left(\mathbf{R}^{d}\right) \text { and } \quad \Sigma_{s}^{\prime}\left(\mathbf{R}^{d}\right)=\bigcup_{h>0} \mathcal{S}_{s, h}^{\prime}\left(\mathbf{R}^{d}\right)
$$

Note that $\mathcal{S}_{S}^{\prime}\left(\mathbf{R}^{d}\right)$ is the dual of $\mathcal{S}_{S}\left(\mathbf{R}^{d}\right)$, and $\Sigma_{S}^{\prime}\left(\mathbf{R}^{d}\right)$ is the dual of $\Sigma_{S}\left(\mathbf{R}^{d}\right)$ as proved in [22].

By the definitions we have

$$
\begin{aligned}
& \mathcal{S}_{1 / 2} \hookrightarrow \Sigma_{s} \hookrightarrow \mathcal{S}_{S} \hookrightarrow \Sigma_{s+\varepsilon} \hookrightarrow \mathscr{S} \\
& \hookrightarrow \mathscr{S}^{\prime} \hookrightarrow \Sigma_{s+\varepsilon}^{\prime} \hookrightarrow \mathcal{S}_{S}^{\prime} \hookrightarrow \Sigma_{s}^{\prime} \hookrightarrow \mathcal{S}_{1 / 2}^{\prime}, \quad s>\frac{1}{2}, \varepsilon>0 .
\end{aligned}
$$


We let $\langle\cdot, \cdot\rangle$ denote the dual form between a topological vector space and its dual. If $z, w \in \mathbf{C}^{d}$, then $\langle z, w\rangle$ is defined by

$$
\langle z, w\rangle=\sum_{j=1}^{d} z_{j} w_{j}, \quad z=\left(z_{1}, \ldots, z_{d}\right) \in \mathbf{C}^{d}, w=\left(w_{1}, \ldots, w_{d}\right) \in \mathbf{C}^{d}
$$

The Fourier transform of $f \in L^{1}\left(\mathbf{R}^{d}\right)$ is given by

$$
(\mathscr{F} f)(\xi)=\widehat{f}(\xi) \equiv(2 \pi)^{-\frac{d}{2}} \int_{\mathbf{R}^{d}} f(x) e^{-i\langle x, \xi\rangle} d x .
$$

The map $\mathscr{F}$ extends uniquely to homeomorphisms on $\mathscr{S}^{\prime}\left(\mathbf{R}^{d}\right), \mathcal{S}_{s}^{\prime}\left(\mathbf{R}^{d}\right)$ and on $\Sigma_{s}^{\prime}\left(\mathbf{R}^{d}\right)$. Furthermore, $\mathscr{F}$ restricts to homeomorphisms on $\mathscr{S}\left(\mathbf{R}^{d}\right), \mathcal{S}_{S}\left(\mathbf{R}^{d}\right), \Sigma_{S}\left(\mathbf{R}^{d}\right)$, and to a unitary operator on $L^{2}\left(\mathbf{R}^{d}\right)$. Similar results hold true for partial Fourier transforms.

For a fixed $\phi \in \mathcal{S}_{S}\left(\mathbf{R}^{d}\right)$ the short-time Fourier transform $V_{\phi} f$ of $f \in \mathcal{S}_{S}^{\prime}\left(\mathbf{R}^{d}\right)$ with respect to the window function $\phi$ is the Gelfand-Shilov distribution on $\mathbf{R}^{2 d}$, defined by

$$
V_{\phi} f(x, \xi)=(2 \pi)^{-\frac{d}{2}}\left\langle f, \overline{\phi(\cdot-x)} e^{-i\langle\cdot, \xi\rangle}\right\rangle
$$

If instead $f \in \Sigma_{s}^{\prime}\left(\mathbf{R}^{d}\right)$ or $f \in \mathscr{S}^{\prime}\left(\mathbf{R}^{d}\right)$, then $\phi$ should belong to $\Sigma_{s}\left(\mathbf{R}^{d}\right)$ or $\mathscr{S}\left(\mathbf{R}^{d}\right)$, respectively. We have

$$
V_{\phi} f(x, \xi) \equiv\left(\mathscr{F}_{2}(U(f \otimes \phi))\right)(x, \xi)=\mathscr{F}(f \overline{\phi(\cdot-x)})(\xi)
$$

where $(U F)(x, y)=F(y, y-x)$. Here $\mathscr{F}_{2} F$ denotes the partial Fourier transform of $F(x, y) \in \mathcal{S}_{S}^{\prime}\left(\mathbf{R}^{2 d}\right)$ with respect to the $y$ variable (see (A.1) in [5]).

In the case $f \in \mathcal{S}_{S}\left(\mathbf{R}^{d}\right), V_{\phi} f$ can be written as

$$
V_{\phi} f(x, \xi)=(2 \pi)^{-\frac{d}{2}} \int f(y) \overline{\phi(y-x)} e^{-i\langle y, \xi\rangle} d y .
$$

The next two propositions characterize Gelfand-Shilov functions and their distributions by suitable estimates on the short-time Fourier transforms of the corresponding distributions. The proofs are omitted since the results are special cases of [27, Theorem 2.7]) and [42, Proposition 2.2].

Proposition 2.2 Let $s, s_{0} \geq \frac{1}{2}$ be such that $s_{0} \leq s$. Also let $\phi \in \mathcal{S}_{s_{0}}\left(\mathbf{R}^{d}\right) \backslash 0$ and $f \in \mathcal{S}_{s_{0}}^{\prime}\left(\mathbf{R}^{d}\right)$. Then the following is true:

(1) $f \in \mathcal{S}_{S}\left(\mathbf{R}^{d}\right)$, if and only if

$$
\left|V_{\phi} f(x, \xi)\right| \lesssim e^{-r\left(|x|^{\frac{1}{s}}+|\xi|^{\frac{1}{s}}\right)}
$$

holds for some $r>0$; 
(2) if in addition $s_{0}>\frac{1}{2}$ and $\phi \in \Sigma_{s_{0}}\left(\mathbf{R}^{d}\right)$, then $f \in \Sigma_{S}\left(\mathbf{R}^{d}\right)$, if and only if (2.10) holds for every $r>0$.

Proposition 2.3 Let $s, s_{0} \geq \frac{1}{2}$ be such that $s_{0} \leq$ s. Also let $\phi \in \mathcal{S}_{s_{0}}\left(\mathbf{R}^{d}\right) \backslash 0$ and $f \in \mathcal{S}_{s_{0}}^{\prime}\left(\mathbf{R}^{d}\right)$. Then the following is true:

(1) $f \in \mathcal{S}_{S}^{\prime}\left(\mathbf{R}^{d}\right)$, if and only if

$$
\left|V_{\phi} f(x, \xi)\right| \lesssim e^{r\left(|x|^{\frac{1}{s}}+|\xi|^{\frac{1}{s}}\right)}
$$

holds for every $r>0$;

(2) if in addition $s_{0}>\frac{1}{2}$ and $\phi \in \Sigma_{s_{0}}\left(\mathbf{R}^{d}\right)$, then $f \in \Sigma_{s}^{\prime}\left(\mathbf{R}^{d}\right)$, if and only if (2.11) holds for some $r>0$.

Remark 2.4 For every $s>0$, the mapping $(f, \phi) \mapsto V_{\phi} f$ is continuous from $\mathcal{S}_{s}\left(\mathbf{R}^{d}\right) \times$ $\mathcal{S}_{S}\left(\mathbf{R}^{d}\right)$ to $\mathcal{S}_{S}\left(\mathbf{R}^{2 d}\right)$ and extends uniquely to continuous mappings from $\mathcal{S}_{S}^{\prime}\left(\mathbf{R}^{d}\right) \times$ $\mathcal{S}_{S}^{\prime}\left(\mathbf{R}^{d}\right)$ to $\mathcal{S}_{s}^{\prime}\left(\mathbf{R}^{2 d}\right)$. The same is true if we replace each $\mathcal{S}_{s}$ by $\mathscr{S}$ or by $\Sigma_{s}$. This is admitted by formula (2.9) (cf. e.g. [35,42]).

\subsection{Modulation Spaces}

We recall that a quasi-norm $\|\cdot\|_{\mathscr{B}}$ of order $r \in(0,1]$ on the vector-space $\mathscr{B}$ is a nonnegative functional on $\mathscr{B}$ which satisfies

$$
\begin{aligned}
\|f+g\|_{\mathscr{B}} & \leq 2^{\frac{1}{r}-1}\left(\|f\|_{\mathscr{B}}+\|g\|_{\mathscr{B}}\right), \quad f, g \in \mathscr{B}, \\
\|\alpha \cdot f\|_{\mathscr{B}} & =|\alpha| \cdot\|f\|_{\mathscr{B}}, \quad \alpha \in \mathbf{C}, \quad f \in \mathscr{B}
\end{aligned}
$$

and

$$
\|f\|_{\mathscr{B}}=0 \quad \Leftrightarrow \quad f=0 .
$$

The vector space $\mathscr{B}$ is called a quasi-Banach space if it is a complete quasi-normed space. If $\mathscr{B}$ is a quasi-Banach space with quasi-norm satisfying (2.12) then by [2,29] there is an equivalent quasi-norm to $\|\cdot\|_{\mathscr{B}}$ which additionally satisfies

$$
\|f+g\|_{\mathscr{B}}^{r} \leq\|f\|_{\mathscr{B}}^{r}+\|g\|_{\mathscr{B}}^{r}, \quad f, g \in \mathscr{B}
$$

From now on we always assume that the quasi-norm of the quasi-Banach space $\mathscr{B}$ is chosen in such a way that both (2.12) and (2.13) hold.

Let $\phi \in \Sigma_{1}\left(\mathbf{R}^{d}\right) \backslash 0, p, q \in(0, \infty]$ and $\omega \in \mathscr{P}_{E}\left(\mathbf{R}^{2 d}\right)$. Then the modulation space $M_{(\omega)}^{p, q}\left(\mathbf{R}^{d}\right)$ is defined as the set of all $f \in \Sigma_{1}^{\prime}\left(\mathbf{R}^{d}\right)$ such that

$$
\|f\|_{M_{(\omega)}^{p, q}} \equiv\left(\int\left(\int\left|V_{\phi} f(x, \xi) \omega(x, \xi)\right|^{p} d x\right)^{q / p} d \xi\right)^{1 / q}<\infty
$$

holds. We set $M_{(\omega)}^{p}=M_{(\omega)}^{p, p}$, and if $\omega=1$, we set $M^{p, q}=M_{(\omega)}^{p, q}$ and $M^{p}=M_{(\omega)}^{p}$. 
We summarize some well-known facts about Modulation spaces in the next proposition. See $[12,21,25,41]$ for the proof. Here the conjugate exponent of $p \in(0, \infty]$ is given by

$$
p^{\prime}= \begin{cases}\infty & \text { when } p \in(0,1] \\ \frac{p}{p-1} & \text { when } p \in(1, \infty) \\ 1 & \text { when } p=\infty\end{cases}
$$

Proposition 2.5 Let $p, q, p_{j}, q_{j}, r \in(0, \infty]$ be such that $r \leq \min (1, p, q), j=1,2$, let $\omega, \omega_{1}, \omega_{2}, v \in \mathscr{P}_{E}\left(\mathbf{R}^{2 d}\right)$ be such that $\omega$ is $v$-moderate, $\phi \in M_{(v)}^{r}\left(\mathbf{R}^{d}\right) \backslash 0$ and let $f \in \Sigma_{1}^{\prime}\left(\mathbf{R}^{d}\right)$. Then the following is true:

(1) $f \in M_{(\omega)}^{p, q}\left(\mathbf{R}^{d}\right)$ if and only if (2.14) holds. Moreover, $M_{(\omega)}^{p, q}$ is a quasi-Banach space under the quasi-norm in (2.14) and a Banach space if $p, q \geq 1$. Different choices of $\phi$ give rise to equivalent (quasi-)norms;

(2) if $p_{1} \leq p_{2}, q_{1} \leq q_{2}$ and $\omega_{2} \lesssim \omega_{1}$, then

$$
\Sigma_{1}\left(\mathbf{R}^{d}\right) \subseteq M_{\left(\omega_{1}\right)}^{p_{1}, q_{1}}\left(\mathbf{R}^{d}\right) \subseteq M_{\left(\omega_{2}\right)}^{p_{2}, q_{2}}\left(\mathbf{R}^{d}\right) \subseteq \Sigma_{1}^{\prime}\left(\mathbf{R}^{d}\right)
$$

(3) if in addition $p, q \geq 1$, then the $L^{2}$-form on $\Sigma_{1}\left(\mathbf{R}^{d}\right)$ extends uniquely to a continuous sesqui-linear form on $M_{(\omega)}^{p, q}\left(\mathbf{R}^{d}\right) \times M_{(1 / \omega)}^{p^{\prime}, q^{\prime}}\left(\mathbf{R}^{d}\right)$. Furthermore, if $p, q<\infty$, then the dual of $M_{(\omega)}^{p, q}\left(\mathbf{R}^{d}\right)$ can be identified by $M_{(1 / \omega)}^{p^{\prime}, q^{\prime}}\left(\mathbf{R}^{d}\right)$ through this form.

\subsection{A Broader Family of Modulation Spaces}

In this subsection we introduce a broader class of modulation spaces by imposing certain types of translation invariant solid BF-space norms on the short-time Fourier transform, cf. [12-16].

Definition 2.6 Let $\mathscr{B} \subseteq L_{l o c}^{r}\left(\mathbf{R}^{d}\right)$ be a quasi-Banach space of order $r \in(0,1]$ which contains $\Sigma_{1}\left(\mathbf{R}^{d}\right)$ with continuous embedding, and let $v_{0} \in \mathscr{P}_{E}\left(\mathbf{R}^{d}\right)$. Then $\mathscr{B}$ is called a translation invariant Quasi-Banach Function space on $\mathbf{R}^{d}$ (with respect to $v_{0}$ ), or invariant $Q B F$ space on $\mathbf{R}^{d}$ of order $r$, if there is a constant $C$ such that:

(1) if $x \in \mathbf{R}^{d}$ and $f \in \mathscr{B}$, then $f(\cdot-x) \in \mathscr{B}$, and

$$
\|f(\cdot-x)\|_{\mathscr{B}} \leq C v_{0}(x)\|f\|_{\mathscr{B}}
$$

(2) if $f, g \in L_{l o c}^{r}\left(\mathbf{R}^{d}\right)$ satisfy $g \in \mathscr{B}$ and $|f| \leq|g|$, then $f \in \mathscr{B}$ and

$$
\|f\|_{\mathscr{B}} \leq C\|g\|_{\mathscr{B}} .
$$

If the weight $v_{0}$ even is an element of $\mathscr{P}_{E, s}\left(\mathbf{R}^{d}\right)\left(\mathscr{P}_{E, s}^{0}\left(\mathbf{R}^{d}\right)\right)$, then we call $\mathscr{B}$ of Definition 2.6 an invariant $Q B F$-space of Roumieu type (Beurling type) of order $r$. 
By Definition 2.6(2) we have $f \cdot h \in \mathscr{B}$ and

$$
\|f \cdot h\|_{\mathscr{B}} \leq C\|f\|_{\mathscr{B}}\|h\|_{L^{\infty}},
$$

when $f \in \mathscr{B}$ and $h \in L^{\infty}$.

The QBF space $\mathscr{B}$ is called is called an invariant $B F$-space (with respect to $v_{0}$ ), if it is of order 1 , is continuously embedded in $\Sigma_{1}^{\prime}\left(\mathbf{R}^{d}\right)$ and the map $(f, \varphi) \mapsto f * \varphi$ is well-defined and continuous from $\mathscr{B} \times L_{\left(v_{0}\right)}^{1}\left(\mathbf{R}^{d}\right)$ to $\mathscr{B}$. Note here that an invariant QBF space of order $r=1$ is a Banach space. Because of condition (2) an invariant BF-space is a solid BF-space in the sense of (A.3) in [13]. For the invariant BF-space $\mathscr{B} \subseteq L_{l o c}^{1}\left(\mathbf{R}^{d}\right)$ with respect to $v_{0}$ we have Minkowski's inequality, i. e.

$$
\|f * \varphi\|_{\mathscr{B}} \leq C\|f\|_{\mathscr{B}}\|\varphi\|_{L_{(v)}^{1}}, \quad f \in \mathscr{B}, \varphi \in L_{\left(v_{0}\right)}^{1}\left(\mathbf{R}^{d}\right)
$$

for some $C>0$ which is independent of $f \in \mathscr{B}$ and $\varphi \in L_{\left(v_{0}\right)}^{1}\left(\mathbf{R}^{d}\right)$.

The following result shows that $v_{0}$ in Definition 2.6 can be replaced by a submultiplicative weight $v$ such that (2.15) is true with $v$ in place of $v_{0}$ and the constant $C=1$, and such that

$$
v(x+y) \leq v(x) v(y), \quad x, y \in \mathbf{R}^{d} .
$$

Proposition 2.7 Let $\mathscr{B}$ be an invariant BF-space on $\mathbf{R}^{d}$ with respect to $v_{0} \in \mathscr{P}_{E}\left(\mathbf{R}^{d}\right)$. Then there is a submultiplicative $v \in \mathscr{P}_{E}\left(\mathbf{R}^{d}\right)$ which satisfies (2.15) and (2.18) with $v$ in place of $v_{0}$, and $C=1$.

\section{Proof Let}

$$
v_{1}(x) \equiv \sup _{f \in \mathscr{B}}\left(\frac{\|f(\cdot-x)\|_{\mathscr{B}}}{\|f\|_{\mathscr{B}}}\right) .
$$

Then

$$
\begin{aligned}
v_{1}(x+y) & =\sup _{f \in \mathscr{B}}\left(\frac{\|f(\cdot-x-y)\|_{\mathscr{B}}}{\|f(\cdot-y)\|_{\mathscr{B}}} \cdot \frac{\|f(\cdot-y)\|_{\mathscr{B}}}{\|f\|_{\mathscr{B}}}\right) \\
& \leq \sup _{f \in \mathscr{B}}\left(\frac{\|f(\cdot-x)\|_{\mathscr{B}}}{\|f\|_{\mathscr{B}}}\right) \cdot \sup _{f \in \mathscr{B}}\left(\frac{\|f(\cdot-y)\|_{\mathscr{B}}}{\|f\|_{\mathscr{B}}}\right)=v_{1}(x) v_{1}(y) .
\end{aligned}
$$

The result now follows by letting

$$
v(x)=\max \left(v_{1}(x), v_{1}(-x)\right) .
$$

From now on it is assumed that $v$ and $v_{j}$ are submultiplicative weights if nothing else is stated. 
Example 2.8 For $p, q \in[1, \infty]$ the space $L_{1}^{p, q}\left(\mathbf{R}^{2 d}\right)$ consists of all $f \in L_{l o c}^{1}\left(\mathbf{R}^{2 d}\right)$ such that

$$
\|f\|_{L_{1}^{p, q}} \equiv\left(\int\left(\int|f(x, \xi)|^{p} d x\right)^{q / p} d \xi\right)^{1 / q}<\infty .
$$

Additionally $L_{2}^{p, q}\left(\mathbf{R}^{2 d}\right)$ is the set of all $f \in L_{l o c}^{1}\left(\mathbf{R}^{2 d}\right)$ such that

$$
\|f\|_{L_{2}^{p, q}} \equiv\left(\int\left(\int|f(x, \xi)|^{q} d \xi\right)^{p / q} d x\right)^{1 / p}<\infty .
$$

Then $L_{1}^{p, q}$ and $L_{2}^{p, q}$ are translation invariant BF-spaces with respect to $v=1$.

Next we define the extended class of modulation spaces, which are of main interest for us.

Definition 2.9 Let $\mathscr{B}$ be a translation invariant QBF-space on $\mathbf{R}^{2 d}, \omega \in \mathscr{P}_{E}\left(\mathbf{R}^{2 d}\right)$, and $\phi \in \Sigma_{1}\left(\mathbf{R}^{d}\right) \backslash 0$. Then the set $M(\omega, \mathscr{B})$ consists of all $f \in \Sigma_{1}^{\prime}\left(\mathbf{R}^{d}\right)$ such that

$$
\|f\|_{M(\omega, \mathscr{B})} \equiv\left\|V_{\phi} f \omega\right\|_{\mathscr{B}}<\infty .
$$

Obviously, we have $M_{(\omega)}^{p, q}\left(\mathbf{R}^{d}\right)=M(\omega, \mathscr{B})$ if $\mathscr{B}=L_{1}^{p, q}\left(\mathbf{R}^{2 d}\right)$, see e.g. (2.14). We remark that many properties of the classical modulation spaces are also true for $M(\omega, \mathscr{B})$. For instance, the definition of $M(\omega, \mathscr{B})$ is independent of the choice of $\phi$ when $\mathscr{B}$ is a Banach space. This statement is formulated in the next proposition. We omit the proof since it can be proved by similar arguments as in Proposition 11.3.2 in [25].

Proposition 2.10 Let $\mathscr{B}$ be an invariant BF-space with respect to $v_{0} \in \mathscr{P}_{E}\left(\mathbf{R}^{2 d}\right)$ and let $\omega, v \in \mathscr{P}_{E}\left(\mathbf{R}^{2 d}\right)$ be such that $\omega$ is $v$-moderate. Also let $M(\omega, \mathscr{B})$ be the same as in Definition 2.9, and let $\phi \in M_{\left(v_{0} v\right)}^{1}\left(\mathbf{R}^{d}\right) \backslash 0$ and $f \in \Sigma_{1}^{\prime}\left(\mathbf{R}^{d}\right)$. Then $f \in M(\omega, \mathscr{B})$ if and only if $V_{\phi} f \cdot \omega \in \mathscr{B}$, and different choices of $\phi$ gives rise to equivalent norms of $M(\omega, \mathscr{B})$.

We refer to [12,15-17,21,25,30,41] for more facts about modulation spaces.

In applications, $\mathscr{B}$ is mostly a mixed quasi-normed Lebesgue space, which is defined next. Let $E=\left\{e_{1}, \ldots, e_{d}\right\}$ be an ordered basis of $\mathbf{R}^{d}$ and let $E^{\prime}=\left\{e_{1}^{\prime}, \ldots, e_{d}^{\prime}\right\}$ be such that

$$
\left\langle e_{j}, e_{k}^{\prime}\right\rangle=2 \pi \delta_{j k}, \quad j, k=1, \ldots, d
$$

Then $E^{\prime}$ is called the dual basis of $E$. The corresponding lattice and dual lattice are

$$
\Lambda_{E}=\left\{j_{1} e_{1}+\cdots+j_{d} e_{d} ;\left(j_{1}, \ldots, j_{d}\right) \in \mathbf{Z}^{d}\right\}
$$


and

$$
\Lambda_{E}^{\prime}=\Lambda_{E^{\prime}}=\left\{\iota_{1} e_{1}^{\prime}+\cdots+\iota_{d} e_{d}^{\prime} ;\left(\iota_{1}, \ldots, \iota_{d}\right) \in \mathbf{Z}^{d}\right\}
$$

We also let $\kappa(E)$ be the parallelepiped spanned by the basis $E$.

We define for each $\boldsymbol{q}=\left(q_{1}, \ldots, q_{d}\right) \in(0, \infty]^{d}$,

$$
\max (\boldsymbol{q})=\max \left(q_{1}, \ldots, q_{d}\right) \text { and } \min (\boldsymbol{q})=\min \left(q_{1}, \ldots, q_{d}\right) .
$$

Definition 2.11 Let $E=\left\{e_{1}, \ldots, e_{d}\right\}$ be an orderd basis of $\mathbf{R}^{d}, \omega$ be a weight on $\mathbf{R}^{d}$, $\boldsymbol{p}=\left(p_{1}, \ldots, p_{d}\right) \in(0, \infty]^{d}$ and $r=\min (1, \boldsymbol{p})$. If $f \in L_{l o c}^{r}\left(\mathbf{R}^{d}\right)$, then

$$
\|f\|_{L_{E,(\omega)}^{p}} \equiv\left\|g_{d-1}\right\|_{L^{p}(\mathbf{R})},
$$

where $g_{k}\left(z_{k}\right), z_{k} \in \mathbf{R}^{d-k}, k=0, \ldots, d-1$, are inductively defined as

$$
g_{0}\left(x_{1}, \ldots, x_{d}\right) \equiv\left|f\left(x_{1} e_{1}+\cdots+x_{d} e_{d}\right) \omega\left(x_{1} e_{1}+\cdots+x_{d} e_{d}\right)\right|
$$

and

$$
g_{k}\left(z_{k}\right) \equiv\left\|g_{k-1}\left(\cdot, z_{k}\right)\right\|_{L^{p_{k}(\mathbf{R})}, \quad k=1, \ldots, d-1 .}
$$

The space $L_{E,(\omega)}^{p}\left(\mathbf{R}^{d}\right)$ consists of all $f \in L_{l o c}^{r}\left(\mathbf{R}^{d}\right)$ such that $\|f\|_{L_{E,(\omega)}^{p}}$ is finite, and is called $E$-split Lebesgue space (with respect to $\boldsymbol{p}$ and $\omega$ ).

Let $E, \boldsymbol{p}$ and $\omega$ be the same as in Definition 2.11. Then the discrete version $\ell_{E,(\omega)}^{p}\left(\Lambda_{E}\right)$ of $L_{E,(\omega)}^{p}\left(\mathbf{R}^{d}\right)$ is the set of all sequences $a=\{a(j)\}_{j \in \Lambda_{E}}$ such that the quasi-norm

$$
\|a\|_{\ell_{E,(\omega)}^{p}} \equiv\left\|f_{a}\right\|_{L_{E,(\omega)}^{p}}, \quad f_{a}=\sum_{j \in \Lambda_{E}} a(j) \chi_{j}
$$

is finite. Here $\chi_{j}$ is the characteristic function of $j+\kappa(E)$. We also set $L_{E}^{p}=L_{E,(\omega)}^{p}$ and $\ell_{E}^{\boldsymbol{p}}=\ell_{E,(\omega)}^{\boldsymbol{p}}$ when $\omega=1$.

Definition 2.12 Let $E$ be an ordered basis of the phase space $\mathbf{R}^{2 d}$. Then $E$ is called weakly phase split if there is a subset $E_{0} \subseteq E$ such that the span of $E_{0}$ equals $\left\{(x, 0) \in \mathbf{R}^{2 d} ; x \in \mathbf{R}^{d}\right\}$ and the span of $E \backslash E_{0}$ equals $\left\{(0, \xi) \in \mathbf{R}^{2 d} ; \xi \in \mathbf{R}^{d}\right\}$.

\subsection{Pilipović Flat Spaces, Modulation Spaces Outside Time-Frequency Analysis and the Bargmann Transform}

Besides the characterization by means of the short-time Fourier transform in Proposition 2.2, Gelfand-Shilov spaces also can be characterized via Hermite function expansions. Recall that the Hermite function of order $\alpha \in \mathbf{N}^{d}$ is given by 


$$
h_{\alpha}(x)=\pi^{-\frac{d}{4}}(-1)^{|\alpha|}\left(2^{|\alpha|} \alpha !\right)^{-\frac{1}{2}} e^{\frac{|x|^{2}}{2}}\left(\partial^{\alpha} e^{-|x|^{2}}\right) .
$$

It is well-known that $\left\{h_{\alpha}\right\}_{\alpha \in \mathbf{N}^{d}}$ provides an orthonormal basis for $L^{2}\left(\mathbf{R}^{d}\right)$ and a basis for $\mathscr{S}\left(\mathbf{R}^{d}\right), \Sigma_{s}\left(\mathbf{R}^{d}\right)$ when $s>\frac{1}{2}, \mathcal{S}_{s}\left(\mathbf{R}^{d}\right)$ when $s \geq \frac{1}{2}$ and their duals.

By [23] we have for $s \geq \frac{1}{2}\left(s>\frac{1}{2}\right)$ that $f \in \mathcal{S}_{s}\left(\mathbf{R}^{d}\right)\left(f \in \Sigma_{s}\left(\mathbf{R}^{d}\right)\right)$, if and only if the coefficients $c_{\alpha}(f)$ in its Hermite series expansion

$$
f=\sum_{\alpha \in \mathbf{N}^{d}} c_{\alpha}(f) h_{\alpha}, \quad c_{\alpha}(f)=\left(f, h_{\alpha}\right)_{L^{2}\left(\mathbf{R}^{d}\right)}
$$

fulfill

$$
\left|c_{\alpha}(f)\right| \lesssim e^{-r|\alpha|^{\frac{1}{2 s}}}
$$

for some $r>0$ (for every $r>0$ ) with convergence in $\mathcal{S}_{S}\left(\mathbf{R}^{d}\right)\left(\Sigma_{S}\left(\mathbf{R}^{d}\right)\right)$. Similarly, $f \in \mathcal{S}_{S}^{\prime}\left(\mathbf{R}^{d}\right)\left(f \in \Sigma_{S}^{\prime}\left(\mathbf{R}^{d}\right)\right)$, if and only if

$$
\left|c_{\alpha}(f)\right| \lesssim e^{r|\alpha|^{\frac{1}{2 s}}}
$$

for every $r>0$ (for some $r>0$ ) with convergence in $\mathcal{S}_{s}^{\prime}\left(\mathbf{R}^{d}\right)\left(\Sigma_{s}^{\prime}\left(\mathbf{R}^{d}\right)\right)$.

In $[19,42]$ various kinds of Fourier-invariant function and distribution spaces are obtained by applying suitable topologies on formal power series expansions. In particular, the Pilipović flat space $\mathcal{H}_{b}\left(\mathbf{R}^{d}\right)$, denoted by $\mathcal{H}_{b_{1}}\left(\mathbf{R}^{d}\right)$ in [42], and its dual $\mathcal{H}_{\mathrm{b}}^{\prime}\left(\mathbf{R}^{d}\right)$, are defined by all formal expansions (2.19) such that

$$
\left|c_{\alpha}(f)\right| \lesssim r^{|\alpha|} \alpha !^{-\frac{1}{2}}
$$

for some $r>0$, respectively

$$
\left|c_{\alpha}(f)\right| \lesssim r^{|\alpha|} \alpha !^{\frac{1}{2}}
$$

for every $r>0$. For $f \in \mathcal{H}_{b}^{\prime}\left(\mathbf{R}^{d}\right)$ and $\phi \in \mathcal{H}_{b}\left(\mathbf{R}^{d}\right)$, we define

$$
(f, \phi)_{L^{2}\left(\mathbf{R}^{d}\right)} \equiv \sum_{\alpha \in \mathbf{N}^{d}} c_{\alpha}(f) \overline{c_{\alpha}(\phi)}
$$

If $\phi, f \in L^{2}\left(\mathbf{R}^{d}\right)$, the pairing $(f, \phi)_{L^{2}\left(\mathbf{R}^{d}\right)}$ agrees with the $L^{2}\left(\mathbf{R}^{d}\right)$ scalar product of those two functions.

We remark that $\mathcal{H}_{b}^{\prime}\left(\mathbf{R}^{d}\right)$ is larger than any Fourier-invariant Gelfand-Shilov distribution space, and $\mathcal{H}_{b}\left(\mathbf{R}^{d}\right)$ is smaller than any Fourier-invariant Gelfand-Shilov space.

We notice that $\mathcal{H}_{b}\left(\mathbf{R}^{d}\right)$ and $\mathcal{H}_{b}^{\prime}\left(\mathbf{R}^{d}\right)$ possess interesting mapping properties under the Bargmann transform. In fact, the Bargmann kernel is given by

$$
\mathfrak{A}_{d}(z, y)=\pi^{-\frac{d}{4}} \exp \left(-\frac{1}{2}\left(\langle z, z\rangle+|y|^{2}\right)+2^{\frac{1}{2}}\langle z, y\rangle\right)
$$


which is analytic in $z$. For fixed $z \in \mathbf{C}^{d}$, the function $y \rightarrow \mathfrak{A}_{d}(z, y)$ belongs to $\mathcal{H}_{b}\left(\mathbf{R}^{d}\right)$. The Bargmann transform $\left(\mathfrak{V}_{d} f\right)(z)$ of $f \in \mathcal{H}_{b}^{\prime}\left(\mathbf{R}^{d}\right)$ is then defined by

$$
\left(\mathfrak{V}_{d} f\right)(z)=\left\langle f, \mathfrak{A}_{d}(z, \cdot)\right\rangle \text {. }
$$

Due to [42] we have that $\mathfrak{V}_{d}$ is bijective between $\mathcal{H}_{b}^{\prime}\left(\mathbf{R}^{d}\right)$ and $A\left(\mathbf{C}^{d}\right)$, the set of all entire functions on $\mathbf{C}^{d}$, and restricts to a bijective map from $\mathcal{H}_{b}\left(\mathbf{R}^{d}\right)$ to

$$
\left\{F \in A\left(\mathbf{C}^{d}\right) ;|F(z)| \lesssim e^{R|z|}, \text { for some } R>0\right\}
$$

Later on we need that the Bargmann and the short-time Fourier transform are linked by the formula

$$
\begin{aligned}
\left(\mathfrak{V}_{d} f\right)(x+i \xi) & =(2 \pi)^{\frac{d}{2}} e^{\frac{1}{2}\left(|x|^{2}+|\xi|^{2}\right)} e^{-i\langle x, \xi\rangle}\left(V_{\phi} f\right)\left(2^{\frac{1}{2}} x,-2^{\frac{1}{2}} \xi\right), \\
\phi(x) & =\pi^{-\frac{d}{4}} e^{-\frac{1}{2}|x|^{2}}, \quad x \in \mathbf{R}^{d}
\end{aligned}
$$

which can be shown by straight-forward computations. By means of the operator

$$
\left(U_{\mathfrak{V}} F\right)(x, \xi)=(2 \pi)^{\frac{d}{2}} e^{\frac{1}{2}\left(|x|^{2}+|\xi|^{2}\right)} e^{-i\langle x, \xi\rangle} F\left(2^{\frac{1}{2}} x,-2^{\frac{1}{2}} \xi\right),
$$

where $F$ is a function or a suitable element of $F \in \mathcal{H}_{b}^{\prime}\left(\mathbf{R}^{d}\right)$ we can write the Bargmann transform as

$$
\left(\mathfrak{V}_{d} f\right)(x+i \xi)=\left(U_{\mathfrak{V}}\left(V_{\phi} f\right)\right)(x, \xi) .
$$

Definition 2.13 Let $\phi$ be as in (2.20), $\omega$ be a weight on $\mathbf{R}^{2 d}, \mathscr{B}$ be an invariant QBFspace with respect to $v \in \mathscr{P}_{E}\left(\mathbf{R}^{2 d}\right)$ on $\mathbf{R}^{2 d} \simeq \mathbf{C}^{d}$ of order $r \in(0,1]$, and let $U_{\mathfrak{V}}$ be given by (2.21). Then

(1) $B(\omega, \mathscr{B})$ consists of all $F \in L_{l o c}^{r}\left(\mathbf{R}^{2 d}\right)=L_{l o c}^{r}\left(\mathbf{C}^{d}\right)$ such that

$$
\|F\|_{B(\omega, \mathscr{B})} \equiv\left\|\left(U_{\mathfrak{V}}^{-1} F\right) \omega\right\|_{\mathscr{B}}<\infty
$$

(2) $A(\omega, \mathscr{B})$ consists of all $F \in A\left(\mathbf{C}^{d}\right) \cap B(\omega, \mathscr{B})$ with topology inherited from $B(\omega, \mathscr{B})$

(3) $M(\omega, \mathscr{B})$ consists of all $f \in \mathcal{H}_{b}^{\prime}\left(\mathbf{R}^{d}\right)$ such that

$$
\|f\|_{M(\omega, \mathscr{B})} \equiv\left\|V_{\phi} f \cdot \omega\right\|_{\mathscr{B}}
$$

is finite.

We observe the smaller restrictions on $\omega$ compared to what is the main stream. For example, in Definition 2.13 it is not assumed that $\omega$ should belong to $\mathscr{P}_{E}\left(\mathbf{R}^{2 d}\right)$ or $\mathscr{P}\left(\mathbf{R}^{2 d}\right)$. We still call $M(\omega, \mathscr{B})$ a modulation space. In contrast to earlier situations, it seems that $M(\omega, \mathscr{B})$ is not invariant under the choice of $\phi$ when $\omega$ fails to belong to $\mathscr{P}_{E}$. For that reason we always assume that $\phi$ is given by (2.20) for such $\omega$. 
We have the following.

Proposition 2.14 Let $\phi$ be as in (2.20), $\omega$ be a weight on $\mathbf{R}^{2 d}$ and let $\mathscr{B}$ be an invariant $Q B F$-space with respect to $v \in \mathscr{P}_{E}\left(\mathbf{R}^{2 d}\right)$. Then the following is true:

(1) the map $\mathfrak{V}_{d}$ is an isometric bijection from $M(\omega, \mathscr{B})$ to $A(\omega, \mathscr{B})$;

(2) if in addition $\mathscr{B}$ is a mixed quasi-normed space of Lebesgue type, then $M(\omega, \mathscr{B})$ and $A(\omega, \mathscr{B})$ are quasi-Banach spaces, which are Banach spaces when $\mathscr{B}$ is a Banach space.

For moderate weights, Proposition 2.14 is proved in $[18,24]$ with some completing arguments given in [33]. For the broader weight class $\mathscr{P}_{Q}$ in Definition 2.1, a proof of Proposition 2.14 is given in [40]. We now present a proof which holds for any weight $\omega$.

Proof From (2.20), (2.21) and Definition 2.13 it follows that $\mathfrak{V}_{d}$ is an isometric injection from $M(\omega, \mathscr{B})$ to $A(\omega, \mathscr{B})$. Since any element in $A\left(\mathbf{C}^{d}\right)$, and thereby any element in $A(\omega, \mathscr{B})$ is a Bargmann transform of an element in $\mathcal{H}_{\mathrm{b}}^{\prime}\left(\mathbf{R}^{d}\right)$, it follows that the image of $M(\omega, \mathscr{B})$ under $\mathfrak{V}_{d}$ contains $A(\omega, \mathscr{B})$. This gives the stated bijectivity in (1).

The completeness of $A(\omega, \mathscr{B})$, and thereby of $M(\omega, \mathscr{B})$ follows from [42, Theorem 4.8]. This gives (2).

\section{Compactness Properties for Modulation Spaces}

This section is devoted to the questions under which sufficient and necessary conditions the inclusion map

$$
i: M\left(\omega_{1}, \mathscr{B}\right) \rightarrow M\left(\omega_{2}, \mathscr{B}\right)
$$

is continuous or even compact for suitable invariant QBF-spaces $\mathscr{B}$.

As ingredients for the proof of our main results we need to deduce some properties for moderate weight functions. In what follows let $L_{0,(\omega)}^{\infty}\left(\mathbf{R}^{d}\right)$ be the set of all $f \in$ $L_{(\omega)}^{\infty}\left(\mathbf{R}^{d}\right)$ with the property

$$
\lim _{R \rightarrow \infty}\left(\operatorname{ess} \sup _{|x| \geq R}|f(x) \omega(x)|\right)=0
$$

when $\omega$ is a weight on $\mathbf{R}^{d}$. We also set $L_{0}^{\infty}=L_{0,(\omega)}^{\infty}$ when $\omega=1$. If $\Lambda$ is a lattice, then the discrete Lebesgue spaces $\ell_{0}^{\infty}(\Lambda)$ and $\ell_{0,(\omega)}^{\infty}(\Lambda)$ are defined analogously.

Lemma 3.1 Let $E$ be an ordered basis of $\mathbf{R}^{d}$ and let $\omega \in \mathscr{P}_{E}\left(\mathbf{R}^{d}\right)$. Then the following is true:

(1) $\mathscr{P}_{E}\left(\mathbf{R}^{d}\right)$ is a convex cone which is closed under multiplication, division and under compositions with power functions;

(2) $\mathscr{P}_{E}\left(\mathbf{R}^{d}\right) \cap L_{E,(\omega)}^{p}\left(\mathbf{R}^{d}\right)$ increases with $\boldsymbol{p} \in(0, \infty]^{d}$, and

$$
\mathscr{P}_{E}\left(\mathbf{R}^{d}\right) \cap L_{E,(\omega)}^{p}\left(\mathbf{R}^{d}\right) \subseteq \mathscr{P}_{E}\left(\mathbf{R}^{d}\right) \cap L_{0,(\omega)}^{\infty}\left(\mathbf{R}^{d}\right), \quad \boldsymbol{p} \in(0, \infty)^{d} .
$$

Similar properties have been shown in [3, Lemma 2.1] for the subset $\mathscr{P}$ of $\mathscr{P}_{E}$. 
Proof Claim (1) can easily be verified by means of the definition of moderate weights. It remains to verify (2). Let $\kappa(E)$ be the (closed) parallelepiped spanned by $E$ and let $\vartheta \in \mathscr{P}_{E}\left(\mathbf{R}^{d}\right)$. By using the map $\vartheta \mapsto \vartheta \cdot \omega$, we reduce ourself to the case when $\omega=1$.

The moderateness of $\vartheta \in \mathscr{P}_{E}\left(\mathbf{R}^{2 d}\right)$ implies that

$$
\vartheta\left(x_{1}\right) \asymp \vartheta\left(x_{1}+x_{2}\right), \text { when } x_{2} \in \kappa(E) .
$$

Hence, if $\chi_{j}$ is the characteristic function of $j+\kappa(E)$ and

$$
\vartheta_{0}(x)=\sum_{j \in \Lambda_{E}} \vartheta(j) \chi_{j}(x)
$$

then $\vartheta \asymp \vartheta_{0}$, giving that

$$
\|\vartheta\|_{L_{E}^{p}} \asymp\left\|\vartheta_{0}\right\|_{L_{E}^{p}} \asymp\|\vartheta\|_{\ell_{E}^{p}}
$$

The assertion now follows from the fact that $\ell_{E}^{\boldsymbol{p}}$ increases with $\boldsymbol{p}$ and that if in addition $\boldsymbol{p} \in(0, \infty)^{d}$, then $\ell_{E}^{\boldsymbol{p}} \subseteq \ell_{0}^{\infty}$.

We also need the following extension of [43, Theorem 2.5].

Proposition 3.2 Let $v, v_{0} \in \mathscr{P}_{E}\left(\mathbf{R}^{2 d}\right)$ be submultiplicative, $\omega \in \mathscr{P}_{E}\left(\mathbf{R}^{2 d}\right)$ be $v$ moderate, and let $\mathscr{B}$ be an invariant $B F$-space with respect to $v_{0}$. Then $M(\omega, \mathscr{B})$ is a Banach space, and

$$
M(\omega, \mathscr{B}) \hookrightarrow M_{\left(1 /\left(v_{0} v\right)\right)}^{\infty}\left(\mathbf{R}^{d}\right) .
$$

Remark 3.3 If $\mathscr{B}=L_{E}^{\boldsymbol{p}}\left(\mathbf{R}^{2 d}\right)$ for some weakly phase split basis $E$ of $\mathbf{R}^{2 d}, \boldsymbol{p} \in$ $(0, \infty]^{2 d}$ and $\omega \in \mathscr{P}_{E}\left(\mathbf{R}^{2 d}\right)$, then $M(\omega, \mathscr{B})$ is a quasi-Banach space. Moreover $M\left(\omega, L_{E}^{p}\left(\mathbf{R}^{2 d}\right)\right)$ is increasing with $\boldsymbol{p}$. In particular, (3.3) is improved in [41] into

$$
M\left(\omega, L_{E}^{p}\left(\mathbf{R}^{2 d}\right)\right) \hookrightarrow M_{(\omega)}^{\infty}\left(\mathbf{R}^{2 d}\right) .
$$

For the proof of Proposition 3.2 we need to consider the twisted convolution, $\widehat{*}$, defined by

$$
(F \widehat{*} G)(x, \xi)=(2 \pi)^{-\frac{d}{2}} \iint_{\mathbf{R}^{2 d}} F(x-y, \xi-\eta) G(y, \eta) e^{-i\langle x-y, \eta\rangle} d y d \eta
$$

when $F, G \in L^{1}\left(\mathbf{R}^{2 d}\right)$. The twisted convolution map $\mathcal{T}$, which takes suitable pairs of functions and distributions $(F, G)$ into $F \widehat{*} G$, is continuous between several function and distribution spaces, see e.g. [25] or Lemma 3 in [7]. For example the map $\mathcal{T}$ is continuous from $L^{1}\left(\mathbf{R}^{2 d}\right) \times L^{1}\left(\mathbf{R}^{2 d}\right)$ to $L^{1}\left(\mathbf{R}^{2 d}\right)$. For any $s>0$ it restricts to continuous mappings

$$
\begin{aligned}
& \mathcal{T}: \mathcal{S}_{s}\left(\mathbf{R}^{2 d}\right) \times \mathcal{S}_{S}\left(\mathbf{R}^{2 d}\right) \rightarrow \mathcal{S}_{s}\left(\mathbf{R}^{2 d}\right), \\
& \mathcal{T}: \Sigma_{S}\left(\mathbf{R}^{2 d}\right) \times \Sigma_{S}\left(\mathbf{R}^{2 d}\right) \rightarrow \Sigma_{s}\left(\mathbf{R}^{2 d}\right),
\end{aligned}
$$


and by duality it follows that these mappings extend to continuous mappings

$$
\begin{aligned}
& \mathcal{T}: \mathcal{S}_{s}^{\prime}\left(\mathbf{R}^{2 d}\right) \times \mathcal{S}_{s}\left(\mathbf{R}^{2 d}\right) \rightarrow \mathcal{S}_{s}^{\prime}\left(\mathbf{R}^{2 d}\right), \\
& \mathcal{T}: \Sigma_{s}^{\prime}\left(\mathbf{R}^{2 d}\right) \times \Sigma_{s}\left(\mathbf{R}^{2 d}\right) \rightarrow \Sigma_{s}^{\prime}\left(\mathbf{R}^{2 d}\right) .
\end{aligned}
$$

In fact, for some map $A_{*}$ we have $A_{*}(F \widehat{*} G)=\left(A_{*} F\right) \circ\left(A_{*} G\right)$. This map $A_{*}$ is similar to the map $A$ in Sections 1 and 2 in [4], and by identifying operators with their kernels, $A_{*}$ consists of pullbacks of partial Fourier transforms and non-degenerate linear transformations on the phase space. Since such pullbacks are homeomorphic on any Fourier invariant Gelfand-Shilov space, (3.4) follows from the fact that the sets

$$
\left\{T_{K} ; K \in \mathcal{S}_{S}\left(\mathbf{R}^{2 d}\right)\right\} \text { and }\left\{T_{K} ; K \in \Sigma_{S}\left(\mathbf{R}^{2 d}\right)\right\}
$$

are algebras under compositions. Here $T_{K}$ denotes the linear operator with distribution kernel $K$.

The twisted convolution is convenient to use when changing window functions in short-time Fourier transforms. In fact by Fourier's inversion formula and some straight-forward computations one has

$$
\left(\phi_{3}, \phi_{1}\right)_{L^{2}} \cdot V_{\phi_{2}} f=\left(V_{\phi_{1}} f\right) \widehat{*}\left(V_{\phi_{2}} \phi_{3}\right)
$$

for every $f \in \Sigma_{S}^{\prime}\left(\mathbf{R}^{d}\right)$ and $\phi_{1}, \phi_{2}, \phi_{3} \in \Sigma_{S}\left(\mathbf{R}^{d}\right)$. For any $\phi \in \Sigma_{S}\left(\mathbf{R}^{d}\right) \backslash 0$, we are also interested of the operator $P_{\phi}$, given by

$$
P_{\phi} F \equiv\|\phi\|_{L^{2}\left(\mathbf{R}^{d}\right)}^{-2} F \widehat{*}\left(V_{\phi} \phi\right), \quad F \in \Sigma_{S}^{\prime}\left(\mathbf{R}^{2 d}\right) .
$$

Since $V_{\phi_{2}} \phi_{3} \in \Sigma_{s}\left(\mathbf{R}^{2 d}\right)$ when $\phi_{2}, \phi_{3} \in \Sigma_{S}\left(\mathbf{R}^{d}\right)$, it follows from the continuity properties of the twisted convolution above that $P_{\phi}$ in (3.7) is continuous on $\Sigma_{s}\left(\mathbf{R}^{2 d}\right)$ and on $\Sigma_{s}^{\prime}\left(\mathbf{R}^{2 d}\right)$. Similar facts hold true with $\mathcal{S}_{s}$ and $\mathcal{S}_{s}^{\prime}$ in place of $\Sigma_{s}$ and $\Sigma_{s}^{\prime}$, respectively, at each place.

The operator $P_{\phi}$ has the following properties.

Lemma 3.4 Let $s>0, \phi \in \Sigma_{s}\left(\mathbf{R}^{d}\right) \backslash 0$. Then the following is true:

(1) $P_{\phi}$ in (3.7) is a continuous projection from $\Sigma_{S}^{\prime}\left(\mathbf{R}^{2 d}\right)$ to

$$
V_{\phi}\left(\Sigma_{s}^{\prime}\left(\mathbf{R}^{d}\right)\right) \equiv\left\{V_{\phi} f ; f \in \Sigma_{s}^{\prime}\left(\mathbf{R}^{d}\right)\right\} \subseteq \Sigma_{s}^{\prime}\left(\mathbf{R}^{2 d}\right) \bigcap C^{\infty}\left(\mathbf{R}^{2 d}\right) ;
$$

(2) $P_{\phi}$ in (3.7) restricts to a continuous projection from $\Sigma_{S}\left(\mathbf{R}^{2 d}\right)$ to

$$
V_{\phi}\left(\Sigma_{s}\left(\mathbf{R}^{d}\right)\right) \equiv\left\{V_{\phi} f ; f \in \Sigma_{S}\left(\mathbf{R}^{d}\right)\right\} \subseteq \Sigma_{S}\left(\mathbf{R}^{2 d}\right)
$$

(3) if $\mathscr{B}$ is an invariant $B F$-space on $\mathbf{R}^{2 d}$, then $P_{\phi}$ is continuous on $\mathscr{B}$.

Similar facts hold true with $\mathcal{S}_{s}$ and $\mathcal{S}_{s}^{\prime}$ in place of $\Sigma_{s}$ and $\Sigma_{s}^{\prime}$, respectively, at each place. 
Related results can essentially be found in e. g. [20,24]. In order to be self-contained, we here give a short proof.

Proof We only prove the result in the Beurling case. The Roumieu case follows by similar arguments and is left for the reader.

By (3.6) it is clear that $P_{\phi}$ is the identity map on $V_{\phi}\left(\Sigma_{s}^{\prime}\left(\mathbf{R}^{d}\right)\right)$ and thereby on $V_{\phi}\left(\Sigma_{s}\left(\mathbf{R}^{d}\right)\right)$.

Let $V_{\phi}^{*}$ be the $L^{2}$-adjoint of $V_{\phi}$. That is, $V_{\phi}^{*} F$ satisfies

$$
\left(V_{\phi}^{*} F, \psi\right)_{L^{2}\left(\mathbf{R}^{d}\right)}=\left(F, V_{\phi} \psi\right)_{L^{2}\left(\mathbf{R}^{2 d}\right)}, \quad F \in \Sigma_{S}^{\prime}\left(\mathbf{R}^{2 d}\right), \psi \in \Sigma_{S}\left(\mathbf{R}^{d}\right) .
$$

By the continuity properties of $V_{\phi}$ on $\Sigma_{s}$ and $\Sigma_{s}^{\prime}$, it follows that $V_{\phi}^{*}$ is continuous from $\Sigma_{s}^{\prime}\left(\mathbf{R}^{2 d}\right)$ to $\Sigma_{s}^{\prime}\left(\mathbf{R}^{d}\right)$ and restricts to a continuous map from $\Sigma_{S}\left(\mathbf{R}^{2 d}\right)$ to $\Sigma_{S}\left(\mathbf{R}^{d}\right)$.

By a straight-forward application of Fourier's inversion formula it follows that

$$
P_{\phi} F=V_{\phi} f \text { when } f=\|\phi\|_{L^{2}}^{-2} V_{\phi}^{*} F
$$

which shows that the images of $\Sigma_{s}^{\prime}\left(\mathbf{R}^{2 d}\right)$ and $\Sigma_{s}\left(\mathbf{R}^{2 d}\right)$ under $P_{\phi}$ equal $V_{\phi}\left(\Sigma_{s}^{\prime}\left(\mathbf{R}^{d}\right)\right)$ and $V_{\phi}\left(\Sigma_{S}\left(\mathbf{R}^{d}\right)\right)$, respectively. This gives (1) and (2).

If $\mathscr{B}$ is an invariant BF-space on $\mathbf{R}^{2 d}$ and $F \in \mathscr{B}$, then it follows from the definitions that

$$
\left|P_{\phi} F\right| \lesssim|F| * \Phi
$$

where $\Phi=\left|V_{\phi} \phi\right|$ belongs to $L_{(v)}^{1}\left(\mathbf{R}^{2 d}\right)$ for every choice of $v \in \mathscr{P}_{E}\left(\mathbf{R}^{2 d}\right)$. Hence, a combination of (2) in Definition 2.6 and (2.17) gives $P_{\phi} F \in \mathscr{B}$ and

$$
\left\|P_{\phi} F\right\|_{\mathscr{B}} \lesssim\|F\|_{\mathscr{B}}\|\Phi\|_{L_{(v)}^{1}}
$$

for some $v \in \mathscr{P}_{E}\left(\mathbf{R}^{2 d}\right)$, and the continuity of $P_{\phi}$ on $\mathscr{B}$ follows. This gives (3).

Lemma 3.5 If $\mathscr{B}$ is an invariant BF-space of $\mathbf{R}^{d}$, then

$$
L_{(v)}^{\infty}\left(\mathbf{R}^{d}\right) \hookrightarrow \mathscr{B}
$$

for some $v \in \mathscr{P}_{E}\left(\mathbf{R}^{d}\right)$.

Proof Since $\Sigma_{1}\left(\mathbf{R}^{d}\right)$ is continuously embedded in $\mathscr{B}$ we have

$$
\|f\|_{\mathscr{B}} \lesssim \sup _{\beta \in \mathbf{N}^{d}}\left(\frac{\left\|\left(D^{\beta} f\right) \cdot e^{|\cdot| / h_{0}}\right\|_{L^{\infty}}}{h_{0}^{|\beta|} \beta !}\right)
$$

for some $h_{0}>0$. Let $\omega=e^{-2|\cdot| / h_{0}}, \omega_{0}=\omega * e^{-|\cdot|^{2} / 2}$ and let $v=1 / \omega_{0}$. Then $\omega \in \mathscr{P}_{E}\left(\mathbf{R}^{d}\right)$, and [1, Proposition 1.6] shows that $\omega_{0} \in \mathscr{P}_{E}\left(\mathbf{R}^{d}\right)$ and that

$$
\left|D^{\beta} \omega_{0}\right| \lesssim h^{|\beta|} \beta ! e^{-2|\cdot| / h_{0}}
$$


holds for every $h>0$. By choosing $h<h_{0}$ we get

$$
\begin{aligned}
&\left\|\omega_{0}\right\|_{\mathscr{B}} \lesssim \sup _{\beta \in \mathbf{N}^{d}}\left(\frac{\left\|\left(D^{\beta} \omega_{0}\right) \cdot e^{|\cdot| / h_{0}}\right\|_{L^{\infty}}}{h_{0}^{|\beta|} \beta !}\right) \lesssim \sup _{\beta \in \mathbf{N}^{d}}\left(\frac{h^{|\beta|} \beta !\left\|\omega_{0} \cdot e^{|\cdot| / h_{0}}\right\|_{L^{\infty}}}{h_{0}^{|\beta|} \beta !}\right) \\
& \lesssim\left\|e^{-2|\cdot| / h_{0}} \cdot e^{|\cdot| / h_{0}}\right\|_{L^{\infty}}<\infty
\end{aligned}
$$

Hence, if $f \in L_{(v)}^{\infty}\left(\mathbf{R}^{d}\right)$, then $|f| \lesssim \omega_{0}$ which implies that $f \in \mathscr{B}$ in view of Definition 2.6(2). Furthermore,

$$
\|f\|_{\mathscr{B}} \lesssim\left\|\omega_{0}\right\|_{\mathscr{B}}\|f \cdot v\|_{L^{\infty}} \asymp\|f\|_{L_{(v)}^{\infty}}
$$

and the result follows.

In what follows we let

$$
\mathscr{B}_{(\omega)} \equiv\left\{f \in L_{l o c}^{1}\left(\mathbf{R}^{d}\right) ; f \cdot \omega \in \mathscr{B}\right\},
$$

where $\mathscr{B}$ be an invariant BF-space on $\mathbf{R}^{d}$.

Lemma 3.6 Let $\mathscr{B}$ be an invariant BF-space on $\mathbf{R}^{d}$ and $\omega \in \mathscr{P}_{E}\left(\mathbf{R}^{d}\right)$. Then $\mathscr{B}_{(\omega)}$ is an invariant $B F$-space under the norm

$$
f \mapsto\|f\|_{\mathscr{B}_{(\omega)}} \equiv\|f \cdot \omega\|_{\mathscr{B}}
$$

Proof It is obvious that $\mathscr{B}_{(\omega)}$ is complete. Let $v$ be as in Lemma 3.5. By Lemma 3.5, $L_{(\omega \cdot v)}^{\infty} \hookrightarrow \mathscr{B}_{(\omega)}$. Since

$$
\Sigma_{1}\left(\mathbf{R}^{d}\right) \hookrightarrow L_{(\omega \cdot v)}^{\infty}\left(\mathbf{R}^{d}\right),
$$

we obtain that $\Sigma_{1}\left(\mathbf{R}^{d}\right)$ is continuously embedded in $\mathscr{B}_{(\omega)}$.

By straight-forward computations it follows that both (1) and (2) in Definition 2.6 are fulfilled with $\mathscr{B}_{(\omega)}$ in place of $\mathscr{B}$ provided $v$ in that definition has been modified in suitable ways.

Proof of Proposition 3.2 Let $\phi \in \Sigma_{1}\left(\mathbf{R}^{d}\right) \backslash 0$ be fixed. Since $\omega$ is a moderate function, it follows by the previous lemma that $\mathscr{B}_{(\omega)}$ is an invariant BF-space.

Let $\left\{f_{j}\right\}_{j=1}^{\infty}$ be a Cauchy sequence in $M(\omega, \mathscr{B})$. Then $\left\{V_{\phi} f_{j}\right\}_{j=1}^{\infty}$ is a Cauchy sequence in $\mathscr{B}_{(\omega)}$. Since $\mathscr{B}_{(\omega)}$ is a Banach space, there is a unique $F \in \mathscr{B}_{(\omega)}$ such that

$$
\lim _{j \rightarrow \infty}\left\|V_{\phi} f_{j}-F\right\|_{\mathscr{B}_{(\omega)}}=0
$$

Let $f=\|\phi\|_{L^{2}}^{-2} V_{\phi}^{*} F$. Then $V_{\phi} f=P_{\phi} F$ belongs to $\mathscr{B}_{(\omega)}$ in view of Lemma 3.4 (3). Since $P_{\phi}$ is continuous on $\mathscr{B}_{(\omega)}$ and satisfies the mapping properties given in Lemma 3.4, we get 


$$
\begin{aligned}
& \lim _{j \rightarrow \infty}\left\|f_{j}-f\right\|_{M(\omega, \mathscr{B})}=\lim _{j \rightarrow \infty}\left\|V_{\phi}\left(f_{j}-f\right)\right\|_{\mathscr{B}_{(\omega)}} \\
& \quad=\lim _{j \rightarrow \infty}\left\|P_{\phi}\left(V_{\phi} f_{j}-F\right)\right\|_{\mathscr{B}_{(\omega)}} \lesssim \lim _{j \rightarrow \infty}\left\|V_{\phi} f_{j}-F\right\|_{\mathscr{B}_{(\omega)}}=0 .
\end{aligned}
$$

Hence, $f_{j} \rightarrow f$ in $M(\omega, \mathscr{B})$, and the completeness of $M(\omega, \mathscr{B})$ follows. Consequently, $M(\omega, \mathscr{B})$ is a Banach space.

The embedding (3.3) is an immediate consequence of [43, Theorem 2.5] and the fact that $M(\omega, \mathscr{B})$ is a Banach space.

If we assume that $\mathscr{B}$ is an invariant QBF-space (instead of invariant BF-space) with respect of $v_{0}$, then it seems to be an open question wether (3.3) might be violated or not.

Before studying compactness of embeddings between modulation spaces, we first consider related continuity questions.

Theorem 3.7 Let $\omega_{1}$ and $\omega_{2}$ be weights on $\mathbf{R}^{2 d}, \mathscr{B}$ be an invariant BF-space on $\mathbf{R}^{2 d}$ with respect to $v \in \mathscr{P}_{E}\left(\mathbf{R}^{2 d}\right)$ or a mixed quasi-normed space of Lebesgue type. Then the following is true:

(1) if $\omega_{2} / \omega_{1} \in L^{\infty}\left(\mathbf{R}^{2 d}\right)$, then $M\left(\omega_{1}, \mathscr{B}\right) \subseteq M\left(\omega_{2}, \mathscr{B}\right)$ and the injection

$$
i: M\left(\omega_{1}, \mathscr{B}\right) \rightarrow M\left(\omega_{2}, \mathscr{B}\right) .
$$

is continuous;

(2) if in addition $\omega_{1}, \omega_{2} \in \mathscr{P}_{E}\left(\mathbf{R}^{2 d}\right)$ and $v$ is bounded, then the map (3.8) is a continuous injection, if and only if $\omega_{2} / \omega_{1} \in L^{\infty}\left(\mathbf{R}^{2 d}\right)$.

The next lemma is related to Remark 3.3 and is needed to verify the previous theorem.

Lemma 3.8 Let $v$ be submultiplicative and bounded on $\mathbf{R}^{2 d}, \mathscr{B}$ be an invariant $B F$ space and let $\omega \in \mathscr{P}_{E}\left(\mathbf{R}^{2 d}\right)$. Then $M(\omega, \mathscr{B}) \hookrightarrow M_{(\omega)}^{\infty}\left(\mathbf{R}^{d}\right)$.

Proof Let $\mathscr{B}^{\prime}$ be the $L^{2}$-dual of $\mathscr{B}$ and let $f \in \Sigma_{1}^{\prime}\left(\mathbf{R}^{d}\right)$. Then it follows by straightforward computations that both $\mathscr{B}$ and $\mathscr{B}^{\prime}$ are translation invariant Banach spaces of order 1 which contain $\Sigma_{1}\left(\mathbf{R}^{2 d}\right)$. Let $\phi \in \Sigma_{1}\left(\mathbf{R}^{d}\right)$ be such that $\|\phi\|_{L^{2}}=1$ and let

$$
\Omega=\left\{g \in \Sigma_{1}\left(\mathbf{R}^{d}\right) ;\|g\|_{M_{(1 / \omega)}^{1}} \leq 1\right\} .
$$

By Feichtinger's minimality principle (cf. the extension [43, Theorem 2.4] of [24, Theorem 12.1.9]) one gets

$$
\left\|V_{\phi} g / \omega\right\|_{\mathscr{B}^{\prime}} \asymp\|g\|_{M\left(1 / \omega, \mathscr{B}^{\prime}\right)} \lesssim\|g\|_{M_{(1 / \omega)}^{1}}<\infty .
$$

Together with Proposition 2.5 we get

$$
\begin{aligned}
& \|f\|_{M_{(\omega)}^{\infty}} \asymp \sup _{g \in \Omega}\left|(f, g)_{L^{2}\left(\mathbf{R}^{d}\right)}\right|=\sup _{g \in \Omega}\left|\left(V_{\phi} f \cdot \omega, V_{\phi} g / \omega\right)_{L^{2}\left(\mathbf{R}^{2 d}\right)}\right| \\
& \leq \sup _{g \in \Omega}\left\|V_{\phi} f \cdot \omega\right\|_{\mathscr{B}}\left\|V_{\phi} g / \omega\right\|_{\mathscr{B}} \lesssim\left\|V_{\phi} f \cdot \omega\right\|_{\mathscr{B}} \asymp\|f\|_{M(\omega, \mathscr{B})} .
\end{aligned}
$$


Proof of Theorem 3.7 Claim (1) is an immediate consequence of the boundedness of $\omega_{2} / \omega_{1}$ and of $\mathscr{B}$ being an invariant BF-space.

Assume instead that the embedding $i$ in (3.8) is continuous and the assumptions of the second claim hold. Claim (2) follows if we prove the boundedness of $\omega_{2} / \omega_{1}$, which we aim to deduce by contradiction. Therefore suppose that $\omega_{2} / \omega_{1}$ is unbounded and $M\left(\omega_{1}, \mathcal{B}\right)$ is continuously embedded in $M\left(\omega_{2}, \mathcal{B}\right)$. Then there is a sequence $\left(x_{k}, \xi_{k}\right) \in$ $\mathbf{R}^{2 d}$ with $\left|\left(x_{k}, \xi_{k}\right)\right| \rightarrow \infty$ when $k \rightarrow \infty$ and such that

$$
\frac{\omega_{2}\left(x_{k}, \xi_{k}\right)}{\omega_{1}\left(x_{k}, \xi_{k}\right)} \geq k \text { for all } k \in \mathbf{N} .
$$

Let $\phi$ be as in (2.20) and set

$$
f_{k}=\frac{1}{\omega_{1}\left(X_{k}\right)} e^{i\left\langle\cdot, \xi_{k}\right\rangle} \phi\left(\cdot-x_{k}\right), \quad X_{k}=\left(x_{k}, \xi_{k}\right) .
$$

Also let $v_{0} \in \mathscr{P}_{E}\left(\mathbf{R}^{2 d}\right)$ be submultiplicative and such that $\omega_{1}$ is $v_{0}$-moderate and that $v_{0} \geq 1$.

By

$$
V_{\phi}\left(e^{i\langle\cdot \xi\rangle} f(\cdot-x)\right)(y, \eta)=e^{i\langle x, \eta-\xi\rangle}\left(V_{\phi} f\right)(y-x, \eta-\xi)
$$

which follows by straight-forward computations, see e.g. [25], we get

$$
\left\|e^{i\langle\cdot, \xi\rangle} f(\cdot-x)\right\|_{M\left(\omega_{1}, \mathscr{B}\right)} \leq C \omega_{1}(x, \xi)\|f\|_{M\left(v_{0}, \mathscr{B}\right)}, \quad f \in M\left(v_{0}, \mathscr{B}\right) .
$$

This gives

$$
\left\|f_{k}\right\|_{M\left(\omega_{1}, \mathscr{B}\right)}=\frac{1}{\omega_{1}\left(X_{k}\right)}\left\|e^{i\left\langle\cdot, \xi_{k}\right\rangle} \phi\left(\cdot-x_{k}\right)\right\|_{M\left(\omega_{1}, \mathscr{B}\right)} \leq C\|\phi\|_{M\left(v_{0}, \mathscr{B}\right)}<\infty,
$$

where $C$ is independent of $k \in \mathbb{N}$. Then the hypothesis provides the boundedness of the sequence $\left\{f_{k}\right\}$ in $M\left(\omega_{2}, \mathscr{B}\right)$.

Since $M\left(\omega_{2}, \mathscr{B}\right) \hookrightarrow M_{\left(\omega_{2}\right)}^{\infty}$ due to Lemma 3.8 we have

$$
\sup _{X \in \mathbf{R}^{2 d}} \omega_{2}(X)\left|\left(V_{\phi} f_{k}\right)(X)\right| \leq C\left\|f_{k}\right\|_{M\left(\omega_{2}, \mathscr{B}\right)} \leq C \text { for all } k \in \mathbb{N}
$$

for some $C>0$. In particular by letting $X=X_{k}$ in (3.11) we obtain

$$
\begin{aligned}
& \frac{\omega_{2}\left(X_{k}\right)}{\omega_{1}\left(X_{k}\right)}=(2 \pi)^{\frac{d}{2}} \frac{\omega_{2}\left(X_{k}\right)}{\omega_{1}\left(X_{k}\right)}\left|\left(V_{\phi} \phi\right)(0)\right| \\
& =(2 \pi)^{\frac{d}{2}} \frac{\omega_{2}\left(X_{k}\right)}{\omega_{1}\left(X_{k}\right)} \mid\left(V_{\phi}\left(e^{i\left\langle\cdot, \xi_{k}\right\rangle} \phi\left(\cdot-x_{k}\right)\right)\left(X_{k}\right) \mid\right. \\
& =(2 \pi)^{\frac{d}{2}} \omega_{2}\left(X_{k}\right)\left|\left(V_{\phi} f_{k}\right)\left(X_{k}\right)\right| \leq C,
\end{aligned}
$$

which contradicts (3.9) and proves the result. 
We have now the following extension of [3, Theorem 1.2], which is our main result.

Theorem 3.9 Let $\omega_{1}, \omega_{2} \in \mathscr{P}_{Q}\left(\mathbf{R}^{2 d}\right), v \in \mathscr{P}_{E}\left(\mathbf{R}^{2 d}\right)$ be submultiplicative, $\mathscr{B}$ be an invariant BF-space on $\mathbf{R}^{2 d}$ with respect to $v$ or a mixed quasi-normed space of Lebesgue type. Then the following is true:

(1) if $\omega_{2} / \omega_{1} \in L_{0}^{\infty}\left(\mathbf{R}^{2 d}\right)$, then the injection (3.8) is compact;

(2) if in addition $\omega_{1}, \omega_{2} \in \mathscr{P}_{E}\left(\mathbf{R}^{2 d}\right)$ and $v$ is bounded, then the injection (3.8) is compact, if and only if $\omega_{2} / \omega_{1} \in L_{0}^{\infty}\left(\mathbf{R}^{2 d}\right)$.

We need the following lemma for the proof.

Lemma 3.10 Let $\mathscr{B}$ be an invariant BF space on $\mathbf{R}^{2 d}, \phi(x)=\pi^{-\frac{d}{4}} e^{-\frac{1}{2} \cdot|x|^{2}}, x \in \mathbf{R}^{d}$, $\omega \in \mathscr{P}_{Q}\left(\mathbf{R}^{2 d}\right)$ and let $\left\{f_{j}\right\}_{j=1}^{\infty} \subseteq \Sigma_{1}^{\prime}\left(\mathbf{R}^{d}\right)$ be a bounded set in $M(\omega, \mathscr{B})$. Then there is a subsequence $\left\{f_{j_{k}}\right\}_{k=1}^{\infty}$ of $\left\{f_{j}\right\}_{j=1}^{\infty}$ such that $\left\{V_{\phi} f_{j_{k}}\right\}_{k=1}^{\infty}$ is locally uniformly convergent.

Proof By the link (2.20) between the Bargmann transform and the Gaussian windowed short-time Fourier transform, the result follows if we prove the assertion with $F_{j}=$ $\mathfrak{V}_{d} f_{j}$ in place of $V_{\phi} f_{j}$. For any $R>0$, let $D_{R}$ be the poly-disc

$$
D_{R} \equiv\left\{(x, \xi) \in \mathbf{R}^{2 d} ; x_{j}^{2}+\xi_{j}^{2}<R^{2}, j=1, \ldots, d\right\}
$$

in $\mathbf{R}^{2 d}$ which we identify with

$$
\left\{x+i \xi \in \mathbf{C}^{d} ; x_{j}^{2}+\xi_{j}^{2}<R^{2}, j=1, \ldots, d\right\}
$$

in $\mathbf{C}^{d}$. By Cantor's diagonalization principle the result follows if we prove that for each $R>0$, there is a subsequence $\left\{f_{j_{k}}\right\}_{k=1}^{\infty}$ of $\left\{f_{j}\right\}_{j=1}^{\infty}$ such that $\left\{F_{j_{k}}\right\}_{k=1}^{\infty}$ is uniformly convergent on $D_{R}$.

By [40, Theorem 3.2], we get the boundedness of $\left\{f_{j}\right\}_{j=1}^{\infty}$ in $M_{\left(\omega_{0}\right)}^{\infty}\left(\mathbf{R}^{d}\right)$ for some choice of $\omega_{0} \in \mathscr{P}_{Q}\left(\mathbf{R}^{2 d}\right)$. Hence, $\left\{V_{\phi} f_{j}\right\}_{j=1}^{\infty}$ and thereby $\left\{F_{j}\right\}_{j=1}^{\infty}$ are locally uniformly bounded on $\mathbf{R}^{2 d}$. In particular,

$$
C_{R} \equiv \sup _{j \geq 1}\left\|F_{j}\right\|_{L^{\infty}\left(D_{2 R}\right)} \quad \text { and } \quad C_{R, \omega_{0}} \equiv \sup _{j \geq 1}\left\|F_{j} \omega_{0}\right\|_{L^{\infty}\left(D_{2 R}\right)}
$$

are finite for every weight $\omega_{0}$ on $\mathbf{C}^{d} \simeq \mathbf{R}^{2 d}$.

By Cauchy's and Taylor's formulae we have

$$
F_{j}(z)=\sum_{\alpha \in \mathbf{N}^{d}} a_{j}(\alpha) z^{\alpha}, \quad z \in D_{R},
$$

where

$$
\left|a_{j}(\alpha)\right| \leq C_{R}(2 R)^{-|\alpha|}, \quad \alpha \in \mathbf{N}^{d} .
$$


In particular, if $\left\{\beta_{l}\right\}_{l=1}^{\infty}$ is an enumeration of $\mathbf{N}^{d}$, then for each $l \geq 1,\left\{a_{j}\left(\beta_{l}\right)\right\}_{j=1}^{\infty}$ is a bounded set in $\mathbf{C}$. Hence, for a subsequence $I_{1}=\left\{k_{1,1}, k_{1,2}, \ldots\right\}$ of $\mathbf{Z}_{+}=\{1,2, \ldots\}$, the limit

$$
\lim _{m \rightarrow \infty} a_{k_{1, m}}\left(\beta_{1}\right)
$$

exists. By induction it follows that for some family of subsequences

$$
I_{N}=\left\{k_{N, 1}, k_{N, 2}, \ldots\right\} \subseteq \mathbf{Z}_{+},
$$

which decreases with $N$, the limit

$$
\lim _{m \rightarrow \infty} a_{k_{N, m}}\left(\beta_{n}\right)
$$

exists for every $n \leq N$.

By Cantor's diagonal principle, there is a subsequence $\left\{j_{k}\right\}_{k=1}^{\infty}$ of $\mathbf{Z}_{+}$and sequence $\{b(\alpha)\}_{\alpha \in \mathbf{N}^{d}}$ such that

$$
\lim _{k \rightarrow \infty} a_{j_{k}}(\alpha)=b(\alpha)
$$

By (3.15) we get

$$
|b(\alpha)| \leq C_{R}(2 R)^{-|\alpha|}
$$

This in turn gives

$$
\sup _{j \geq 1}\left\|a_{j}(\alpha) z^{\alpha}\right\|_{L^{\infty}\left(D_{R}\right)} \leq C_{R} 2^{-|\alpha|} \text { and }\left\|b(\alpha) z^{\alpha}\right\|_{L^{\infty}\left(D_{R}\right)} \leq C_{R} 2^{-|\alpha|}
$$

Hence, (3.14) and the Taylor series

$$
F(z) \equiv \sum_{\alpha \in \mathbf{N}^{d}} b(\alpha) z^{\alpha}
$$

are uniformly convergent on $D_{R}$, and by using (3.16), we obtain by straight-forward computations that $F_{j_{k}}$ tends to $F$ uniformly on $D_{R}$ when $k$ tends to infinity.

Proof of Theorem 3.9 In order to verify (1) we need to show that a bounded sequence $\left\{f_{j}\right\}_{j=1}^{\infty}$ in $M\left(\omega_{1}, \mathscr{B}\right)$ has a convergent subsequence in $M\left(\omega_{2}, \mathscr{B}\right)$. By means of the assumptions there is a sequence of increasing balls $B_{k}, k \in \mathbf{Z}_{+}$, centered at the origin with radius tending to $+\infty$ as $k \rightarrow \infty$ such that

$$
\frac{\omega_{2}(x, \xi)}{\omega_{1}(x, \xi)} \leq \frac{1}{k}, \quad \text { when } \quad(x, \xi) \in \mathbf{R}^{2 d} \backslash B_{k} .
$$


By Lemma 3.10 it follows that if $\phi(x)=\pi^{-\frac{d}{4}} e^{-\frac{1}{2} \cdot|x|^{2}}, x \in \mathbf{R}^{d}$, then there is a subsequence $\left\{h_{j}\right\}_{j=1}^{\infty}$ of $\left\{f_{j}\right\}_{j=1}^{\infty}$ such that $\left\{V_{\phi} h_{j}\right\}_{j=1}^{\infty}$ converges uniformly on any $B_{k}$, and converges on the whole $\mathbf{R}^{2 d}$.

Claim (1) follows if we prove $\left\|h_{m_{1}}-h_{m_{2}}\right\|_{M\left(\omega_{2}, \mathscr{B}\right)} \rightarrow 0$ as $m_{1}, m_{2} \rightarrow \infty$. Let $\chi_{k}$ be the characteristic function of $B_{k}, k \geq 1$. From the fact that $C=C_{R}$ in (3.13) is bounded we have

$$
\begin{aligned}
& \left\|h_{m_{1}}-h_{m_{2}}\right\|_{M\left(\omega_{2}, \mathscr{B}\right)}=\left\|V_{\phi} h_{m_{1}}-V_{\phi} h_{m_{2}}\right\|_{\mathscr{B}_{\left(\omega_{2}\right)}} \\
& \quad \lesssim\left\|\left(V_{\phi} h_{m_{1}}-V_{\phi} h_{m_{2}}\right) \chi_{k}\right\|_{\mathscr{B}_{\left(\omega_{2}\right)}}+\left\|V_{\phi} h_{m_{1}}-V_{\phi} h_{m_{2}}\right\|_{\mathscr{B}_{\left(\omega_{1}\right)}} / k \\
& \quad \leq\left\|\left(V_{\phi} h_{m_{1}}-V_{\phi} h_{m_{2}}\right) \chi_{k}\right\|_{\mathscr{B}_{\left(\omega_{2}\right)}}+2 C / k .
\end{aligned}
$$

In order to make the right-hand side arbitrarily small, $k$ is first chosen large enough. Note that $V_{\phi} h_{1}, V_{\phi} h_{2}, \ldots$ is a sequence of bounded continuous functions converging uniformly on the compact set $\bar{B}_{k}$. Since $\omega_{2}$ is a weight and $\mathscr{B}$ is an invariant BF-space we obtain that

$$
\begin{aligned}
& \left\|\left(V_{\phi} h_{m_{1}}-V_{\phi} h_{m_{2}}\right) \chi_{k}\right\|_{\mathscr{B}_{\left(\omega_{2}\right)}}=\left\|\left(V_{\phi} h_{m_{1}}-V_{\phi} h_{m_{2}}\right) \omega_{2} \chi_{k}\right\|_{\mathscr{B}} \\
& \quad \lesssim\left(\sup _{(x, \xi) \in B_{k}}\left|\left(V_{\phi} h_{m_{1}}(x, \xi)-V_{\phi} h_{m_{2}}(x, \xi)\right) \omega_{2}(x, \xi)\right|\right)\left\|\chi_{k}\right\|_{\mathscr{B}} \\
& \quad \lesssim \sup _{(x, \xi) \in B_{k}}\left|V_{\phi} h_{m_{1}}(x, \xi)-V_{\phi} h_{m_{2}}(x, \xi)\right|
\end{aligned}
$$

tends to zero as $m_{1}$ and $m_{2}$ tend to infinity. This proves (1).

In order to verify (2) we suppose that the embedding $i$ in (3.8) is compact and all assumptions of the second claim hold. From the first part of the proof, the result follows if we prove that $\omega_{2} / \omega_{1}$ turns to zero at infinity. We prove this claim by contradiction.

Suppose there is a sequence $\left(x_{k}, \xi_{k}\right) \in \mathbf{R}^{2 d}$ with $\left|\left(x_{k}, \xi_{k}\right)\right| \rightarrow \infty$ if $k \rightarrow \infty$ and a $C>0$ fulfilling

$$
\frac{\omega_{2}\left(x_{k}, \xi_{k}\right)}{\omega_{1}\left(x_{k}, \xi_{k}\right)} \geq C \text { for all } k \in \mathbf{N} .
$$

Let $\psi \in \Sigma_{1}\left(\mathbf{R}^{d}\right), \phi$ be as in (2.20) and let $\left\{f_{k}\right\}_{k=1}^{\infty}$ be as in (3.10).

By the proof of Theorem 3.7, we get the boundedness of $\left\{f_{k}\right\}_{k=1}^{\infty}$ in $M\left(\omega_{1}, \mathscr{B}\right)$, and by the assumptions, $\left\{f_{k}\right\}_{k=1}^{\infty}$ is precompact in $M\left(\omega_{2}, \mathscr{B}\right)$.

Since $\phi \in \Sigma_{1}\left(\mathbf{R}^{d}\right)$ we have $\left|V_{\phi} \psi\right| \lesssim e^{-r|\cdot|}$ for every $r>0$ by Proposition 2.2(2). From the fact $\omega_{1} \gtrsim e^{-r_{0}|\cdot|}$ for some $r_{0}>0$ we get

$$
\int \psi(x) \overline{f_{k}(x)} d x=\frac{1}{\omega_{1}\left(x_{k}, \xi_{k}\right)}\left(V_{\phi} \psi\right)\left(x_{k}, \xi_{k}\right) \rightarrow 0,
$$

as $k \rightarrow \infty$, which implies that $f_{k}$ tends to zero in $\Sigma_{1}^{\prime}\left(\mathbf{R}^{d}\right)$. Hence the only possible limit point in $M\left(\omega_{2}, \mathscr{B}\right)$ of $\left\{f_{k}\right\}_{k=1}^{\infty}$ is zero.

As $\left\{f_{k}\right\}_{k=1}^{\infty}$ is precompact in $M\left(\omega_{2}, \mathscr{B}\right)$, we can extract a subsequence $\left\{f_{k_{j}}\right\}_{j=1}^{\infty}$ which converges to zero in $M\left(\omega_{2}, \mathscr{B}\right)$. 
Since $M\left(\omega_{2}, \mathscr{B}\right) \hookrightarrow M_{\left(\omega_{2}\right)}^{\infty}$ due to Lemma 3.8 we have

$$
\sup _{X \in \mathbf{R}^{2 d}} \omega_{2}(X)\left|\left(V_{\phi}\left(f_{k_{j}}\right)\right)(X)\right| \leq C\left\|f_{k_{j}}\right\|_{M\left(\omega_{2}, \mathscr{B}\right)} \rightarrow 0
$$

as $j \rightarrow \infty$. Taking $X=X_{k_{j}}$ in the previous inequality provides

$$
\begin{aligned}
& \frac{\omega_{2}\left(X_{k_{j}}\right)}{\omega_{1}\left(X_{k_{j}}\right)}=(2 \pi)^{\frac{d}{2}} \frac{\omega_{2}\left(X_{k_{j}}\right)}{\omega_{1}\left(X_{k_{j}}\right)}\left|\left(V_{\phi} \phi\right)(0)\right| \\
& =(2 \pi)^{\frac{d}{2}} \frac{\omega_{2}\left(X_{k_{j}}\right)}{\omega_{1}\left(X_{k_{j}}\right)} \mid\left(V_{\phi}\left(e^{i\left\langle\cdot \xi_{k_{j}}\right\rangle} \phi\left(\cdot-x_{k_{j}}\right)\right)\left(X_{k_{j}}\right) \mid\right. \\
& =(2 \pi)^{\frac{d}{2}} \omega_{2}\left(X_{k_{j}}\right)\left|\left(V_{\phi}\left(f_{k_{j}}\right)\right)\left(X_{k_{j}}\right)\right| \rightarrow 0,
\end{aligned}
$$

which contradicts (3.19) and proves (2).

As an immediate consequence of Lemma 3.1 and Theorem 3.9 we get the following.

Corollary 3.11 Assume that $\omega_{1}, \omega_{2} \in \mathscr{P}_{E}\left(\mathbf{R}^{2 n}\right)$, and that $p, p_{0}, q, q_{0} \in(0, \infty]$ are such that $p_{0}, q_{0}<\infty$. Also assume that $\omega_{2} / \omega_{1} \in L^{p_{0}, q_{0}}\left(\mathbf{R}^{2 d}\right)$. Then the embedding (3.8) is compact.

\section{Applications and Open Questions}

Compactness is a fundamental property in analysis, science and engineering, as remarked in the introduction. In this section we make a review on how the compactness results from the previous section are applied in [1] to deduce index and lifting results for pseudo-differential operators and Toeplitz operators. Thereafter we give some links on some open questions and further developments.

\subsection{Applications to Index Results for Pseudo-Differential and Toeplitz Operators}

Let $A$ be a real $d \times d$ matrix and $\phi \in \Sigma_{1}\left(\mathbf{R}^{d}\right) \backslash 0$ be fixed, and let $a \in \Sigma_{1}\left(\mathbf{R}^{2 d}\right)$. We recall that the pseudo-differential operator $\operatorname{Op}_{A}(a)$ and the Toeplitz operator $\operatorname{Tp}_{\phi}(a)$ are the linear and continuous operators from $\Sigma_{1}\left(\mathbf{R}^{d}\right)$ to $\Sigma_{1}^{\prime}\left(\mathbf{R}^{d}\right)$, defined by the formulae

$$
\left(\mathrm{Op}_{A}(a) f\right)(x)=(2 \pi)^{-d} \iint_{\mathbf{R}^{2 d}} a(x-A(x-y), \xi) f(y) e^{i\langle x-y, \xi\rangle} d y d \xi
$$

and

$$
\left(\operatorname{Tp}_{\phi}(a) f, g\right)_{L^{2}\left(\mathbf{R}^{d}\right)}=\left(a \cdot V_{\phi} f, V_{\phi} g\right)_{L^{2}\left(\mathbf{R}^{2 d}\right)}, \quad f, g \in \Sigma_{1}\left(\mathbf{R}^{d}\right) .
$$

(Cf. [1,5,7,25,28,32,34-40,43,44].) The definitions of pseudo-differential and Toeplitz operators extend in different ways. For example, let $p_{1}$ and $p_{2}$ be positive polynomials on $\mathbf{R}^{d}$ of degrees $n_{1}$ and $n_{2}$, and let 


$$
\omega_{0}(x, \xi)=p_{1}(x)^{r_{1}}+p_{2}(\xi)^{\rho_{1}}
$$

or

$$
\omega_{0}(x, \xi)=\exp \left(\left(p_{1}(x)^{r_{2}}+p_{2}(\xi)^{\rho_{2}}\right)^{r}\right)
$$

for some $r, r_{j}, \rho_{j}>0$ for $j=1,2$, which satisfy

$$
r \cdot \max \left(r_{2} n_{1}, \rho_{2} n_{2}\right)<1
$$

If $\omega \in \mathscr{P}_{E}^{0}\left(\mathbf{R}^{2 d}\right)$ and $p, q \in(0, \infty$ ], then it is proved in [44] that the definition of $\mathrm{Op}_{A}(a)$ above is uniquely extended in such ways that

$$
\mathrm{Op}_{A}\left(\omega_{0}\right): M_{(\omega)}^{p, q}\left(\mathbf{R}^{d}\right) \rightarrow M_{\left(\omega / \omega_{0}\right)}^{p, q}\left(\mathbf{R}^{d}\right)
$$

is continuous. In $[1$, Section 6$]$ it is also proved that

$$
\mathrm{Op}_{A}\left(\omega_{0}\right)-\operatorname{Tp}_{\phi}\left(\omega_{0}\right): M_{(\omega)}^{p, q}\left(\mathbf{R}^{d}\right) \rightarrow M_{\left(\omega / \omega_{1}\right)}^{p, q}\left(\mathbf{R}^{d}\right)
$$

is continuous for some $\omega_{1}$ such that $\omega_{1} / \omega_{0}$ tends to zero at infinity. Finally, in [1, Section 5] it is also proved that

$$
\operatorname{Tp}_{\phi}\left(\omega_{0}\right): M_{(\omega)}^{p, q}\left(\mathbf{R}^{d}\right) \rightarrow M_{\left(\omega / \omega_{0}\right)}^{p, q}\left(\mathbf{R}^{d}\right)
$$

is a continuous bijection with continuous inverse. A combination of (4.4), the fact that $\omega_{1} / \omega_{0}$ tends to zero at infinity and Theorem 3.9 then shows that

$$
\mathrm{Op}_{A}\left(\omega_{0}\right)-\mathrm{Tp}_{\phi}\left(\omega_{0}\right): M_{(\omega)}^{p, q}\left(\mathbf{R}^{d}\right) \rightarrow M_{\left(\omega / \omega_{0}\right)}^{p, q}\left(\mathbf{R}^{d}\right)
$$

is compact.

In particular, if in addition $p, q \geq 1$, then the involved modulation spaces are Banach spaces. Hence, Fredholm's theorem shows that the indices of the operators in (4.3) and (4.5) satisfy

$$
\operatorname{Ind}\left(\mathrm{Op}_{A}\left(\omega_{0}\right)\right)=\operatorname{Ind}\left(\operatorname{Tp}_{\phi}\left(\omega_{0}\right)\right)=0
$$

Here the last equality follows from the fact that (4.5) is a continuous bijection.

If $\omega_{0}$ is given by (4.1), then it follows by straight-forward computations that $\mathrm{Op}_{A}\left(\omega_{0}\right)$ is injective. Since $\operatorname{Ind}\left(\mathrm{Op}_{A}\left(\omega_{0}\right)\right)=0$, it follows that the map (4.3) in this case is bijective.

\subsection{Open Questions and Further Developments}

The main objective in the paper is Theorem 3.9 which completely characterizes compactness for the injection map (3.8) when $\mathscr{B}$ is either an invariant BF-space or a mixed 
quasi-normed space of Lebesgue type, and $\omega_{1}, \omega_{2} \in \mathscr{P}_{E}\left(\mathbf{R}^{2 d}\right)$. An open question here concerns wether such characterizations can be deduced when $\omega_{1}$ and $\omega_{2}$ are allowed to belong to a broader weight class than $\mathscr{P}_{E}\left(\mathbf{R}^{2 d}\right)$.

In fact, for general weights, Theorem 3.9 gives some sufficient but no necessary conditions for the map (3.8) to be compact.

An other open question concerns wether Theorem 3.9 holds for any QBF-space $\mathscr{B}$ with respect to $v=1$ and not only when $\mathscr{B}$ is either an invariant BF-space or a mixed quasi-normed space of Lebesgue type.

Open Access This article is distributed under the terms of the Creative Commons Attribution 4.0 International License (http://creativecommons.org/licenses/by/4.0/), which permits unrestricted use, distribution, and reproduction in any medium, provided you give appropriate credit to the original author(s) and the source, provide a link to the Creative Commons license, and indicate if changes were made.

\section{References}

1. Abdeljawad, A., Coriasco, S., Toft, J.: Liftings for ultra-modulation spaces, and one-parameter groups of Gevrey type pseudo-differential operators. arXiv:1712.04338

2. Aoki, T.: Locally bounded linear topological spaces. Proc. Imp. Acad. Tokyo 18, 588-594 (1942)

3. Boggiatto, P., Toft, J.: Embeddings and compactness for generalized Sobolev-Shubin spaces and modulation spaces. Appl. Anal. 84, 269-282 (2005)

4. Chen, Y., Toft, J.: Boundedness of Gevrey and Gelfand-Shilov kernels of positive semi-definite operators. J. Pseudo-Differ. Oper. Appl. 6, 153-185 (2015)

5. Chen, Y., Toft, J., Wahlberg, P.: The Weyl product on quasi-Banach modulation spaces. Bull. Math. Sci. (Online 2018)

6. Cordero, E., Nicola, F., Rodino, L.: Gabor representations of evolution operators. Trans. Am. Math. Soc. 367, 7639-7663 (2015)

7. Coriasco, S., Johansson, K., Toft, J.: Local wave-front sets of Banach and Fréchet types, and pseudodifferential operators. Monatsh. Math. 169, 285-316 (2013)

8. de Gosson, M., Luef, F.: Born-Jordan pseudodifferential calculus, Bopp operators and deformation quantization. Integr. Equ. Oper. Theory 84, 463-485 (2016)

9. Dörfler, M., Feichtinger, H.G., Gröchenig, K.: Compactness criteria in function spaces. Colloq. Math. 94, 37-50 (2002)

10. Feichtinger, H.G.: Banach spaces of distributions of Wiener's type and interpolation. In: Butzer, P., Nagy, B.S., Görlich, E. (eds.) Proceedings Conference Oberwolfach, Functional Analysis and Approximation, August 1980, International Series of Numerical Mathematics, vol. 69, pp. 153-165. Birkhäuser Verlag, Basel (1981)

11. Feichtinger, H.G.: Banach convolution algebras of Wiener's type. In: Bolyai, J. (eds.) Proceedings Functions, Series, Operators in Budapest, Colloquia Mathematical Society. North Holland Publ. Co., Amsterdam (1980)

12. Feichtinger, H.G.: Modulation spaces on locally compact Abelian groups. Technical report, University of Vienna, Vienna (1983); also in: Krishna, M., Radha, R., Thangavelu, S. (eds.) Wavelets and Their Applications, pp. 99-140. Allied Publishers Private Limited, New Delhi (2003)

13. Feichtinger, H.G.: Wiener amalgams over Euclidean spaces and some of their applications. In: Function Spaces (Edwardsville, IL, 1990), Lecture Notes in Pure and Applied Mathematics, vol. 136, pp. 123137. Marcel Dekker, New York (1992)

14. Feichtinger, H.G.: Modulation spaces: Looking back and ahead. Sampl. Theory Signal Image Process. 5, 109-140 (2006)

15. Feichtinger, H.G., Gröchenig, K.H.: Banach spaces related to integrable group representations and their atomic decompositions, I. J. Funct. Anal. 86, 307-340 (1989) 
16. Feichtinger, H.G., Gröchenig, K.H.: Banach spaces related to integrable group representations and their atomic decompositions, II. Monatsh. Math. 108, 129-148 (1989)

17. Feichtinger, H.G., Gröchenig, K.H.: Gabor frames and time-frequency analysis of distributions. J. Funct. Anal. 2(146), 464-495 (1997)

18. Feichtinger, H.G., GrÃúchenig, K.H., Walnut, D.: Wilson bases and modulation spaces. Math. Nachr. 155, 7-17 (1992)

19. Fernandez, C., Galbis-Verdu, A., Toft, J.: The Bargmann transform and powers of harmonic oscillator on Gelfand-Shilov subspaces. RACSAM 111, 1-13 (2017)

20. Folland, G.B.: Harmonic Analysis in Phase Space. Princeton University Press, Princeton (1989)

21. Galperin, Y.V., Samarah, S.: Time-frequency analysis on modulation spaces $M_{m}^{p, q}, 0<p, q \leq \infty$. Appl. Comput. Harmon. Anal. 16, 1-18 (2004)

22. Gelfand, I.M., Shilov, G.E.: Generalized Functions, I-III. Academic Press, New York (1968)

23. Gramchev, T., Lecke, A., Pilipović, S., Rodino, L.: Gelfand-Shilov type spaces through Hermite expansions. In: Pilipović, S., Toft, J. (eds.) Pseudo-Differential Operators and Generalized Functions, Operator Theory: Advances and Applications, vol. 245, pp. 95-105. Birkhäuser, Basel (2015)

24. Gröchenig, K.H.: Describing functions: atomic decompositions versus frames. Monatsh. Math. 112, 1-42 (1991)

25. Gröchenig, K.H.: Foundations of Time-Frequency Analysis. Birkhäuser, Boston (2001)

26. Gröchenig K.H.: Weight functions in time-frequency analysis. In: Rodino, L., Wong, M.W. (eds.) Pseudodifferential Operators: Partial Differential Equations and Time-Frequency Analysis, Fields Institute Communications, vol. 52, pp. 343-366. American Mathematical Society, Providence, RI (2007)

27. Gröchenig, K.H., Zimmermann, G.: Spaces of test functions via the STFT. J. Funct. Spaces Appl. 2, 25-53 (2004)

28. Hörmander, L.: The Analysis of Linear Partial Differential Operators, vols. I, III, Springer, Berlin $(1983,1985)$

29. Rolewicz, S.: On a certain class of linear metric spaces. Bull. Acad. Polon. Sci. Sér. Sci. Math. Astron. Phys. 5, 471-473 (1957)

30. Ruzhansky, M., Sugimoto, M., Tomita, N., Toft, J.: Changes of variables in modulation and Wiener amalgam spaces. Preprint arXiv:0803.3485v1 (2008)

31. Ruzhansky, M., Sugimoto, M., Wang, B.: Modulation spaces and nonlinear evolution equations. In: Ruzhansky, M., Sugimoto, M., Wirth, J. (eds.) Evolution Equations of Hyperbolic and Schrödinger Type. Progress in Mathematics, vol. 301, pp. 267-283. Birkhäuser/Springer, Basel (2012)

32. Shubin, M.A.: Pseudodifferential Operators and Spectral Theory. Nauka, Moscow (1978) (Russian), Springer (1987) (2001) (English)

33. Signahl, M., Toft, J.: Mapping properties for the Bargmann transform on modulation spaces. J. PseudoDiffer. Oper. Appl. 3, 1-30 (2012)

34. Strohmer, T.: Pseudodifferential operators and Banach algebras in mobile communications. Appl. Comput. Harmon. Anal. 20, 237-249 (2006)

35. Teofanov, N.: Modulation spaces, Gelfand-Shilov spaces and pseudodifferential operators. Sampl. Theory Signal Image Process. 5, 225-242 (2006)

36. Toft, J.: Continuity and Positivity Problems in Pseudo-Differential Calculus. Thesis, Department of Mathematics, University of Lund, Lund (1996)

37. Toft, J.: Subalgebras to a Wiener type algebra of pseudo-differential operators. Ann. Inst. Fourier 51, 1347-1383 (2001)

38. Toft, J.: Continuity properties for modulation spaces with applications to pseudo-differential calculus, I. J. Funct. Anal. 207, 399-429 (2004)

39. Toft, J.: Continuity properties for modulation spaces with applications to pseudo-differential calculus, II. Ann. Global Anal. Geom. 26, 73-106 (2004)

40. Toft, J.: The Bargmann transform on modulation and Gelfand-Shilov spaces, with applications to Toeplitz and pseudo-differential operators. J. Pseudo-Differ. Oper. Appl. 3, 145-227 (2012)

41. Toft, J.: Gabor analysis for a broad class of quasi-Banach modulation spaces. In: Pilipović, S., Toft, J. (eds.) Pseudo-Differential Operators, Generalized Functions, Operator Theory: Advances and Applications, vol. 245, pp. 249-278. Birkhäuser, Basel (2015)

42. Toft, J.: Images of function and distribution spaces under the Bargmann transform. J. Pseudo-Differ. Oper. Appl. 8, 83-139 (2017)

43. Toft, J.: Schatten properties, nuclearity and minimality of phase shift invariant spaces. Appl. Comput. Harmon. Anal. 46, 154-176 (2019) 
44. Toft, J.: Continuity of Gevrey-Hörmander pseudo-differential operators on modulation spaces. J. Pseudo-Differ. Oper. Appl. (Online 2019)

Publisher's Note Springer Nature remains neutral with regard to jurisdictional claims in published maps and institutional affiliations. 